\title{
Claim games for estate division problems
}

Citation for published version (APA):

Peters, H. J. M., Schröder, M. J. W., \& Vermeulen, A. J. (2013). Claim games for estate division problems. Maastricht University, Graduate School of Business and Economics. GSBE Research Memoranda No. 055 https://doi.org/10.26481/umagsb.2013055

Document status and date:

Published: 01/01/2013

DOI:

10.26481/umagsb.2013055

Document Version:

Publisher's PDF, also known as Version of record

\section{Please check the document version of this publication:}

- A submitted manuscript is the version of the article upon submission and before peer-review. There can be important differences between the submitted version and the official published version of record.

People interested in the research are advised to contact the author for the final version of the publication, or visit the DOI to the publisher's website.

- The final author version and the galley proof are versions of the publication after peer review.

- The final published version features the final layout of the paper including the volume, issue and page numbers.

Link to publication

\footnotetext{
General rights rights.

- You may freely distribute the URL identifying the publication in the public portal. please follow below link for the End User Agreement:

www.umlib.nl/taverne-license

Take down policy

If you believe that this document breaches copyright please contact us at:

repository@maastrichtuniversity.nl

providing details and we will investigate your claim.
}

Copyright and moral rights for the publications made accessible in the public portal are retained by the authors and/or other copyright owners and it is a condition of accessing publications that users recognise and abide by the legal requirements associated with these

- Users may download and print one copy of any publication from the public portal for the purpose of private study or research.

- You may not further distribute the material or use it for any profit-making activity or commercial gain

If the publication is distributed under the terms of Article $25 \mathrm{fa}$ of the Dutch Copyright Act, indicated by the "Taverne" license above, 


\section{Maastricht University}

Hans Peters, Marc Schröder, Dries Vermeulen

Claim games for estate division problems

$\mathrm{RM} / 13 / 055$

\section{GSBE}

Maastricht University School of Business and Economics

Graduate School of Business and Economics

P.O Box 616

NL-6200 MD Maastricht

The Netherlands 


\title{
Claim games for estate division problems
}

\author{
Hans Peters*, Marc Schröder*, Dries Vermeulen*
}

This version, August 2013

\begin{abstract}
This paper considers the estate division problem from a non-cooperative perspective. The integer claim game initiated by O'Neill (1982) and extended by Atlamaz et al. (2011) is generalized by considering different sharing rules to divide every interval among the claimants. For problems with an estate larger than half of the total entitlements, we show that every sharing rule satisfying four fairly general axioms yields the same set of Nash equilibrium profiles and corresponding payoffs. Every rule that always results in such equilibrium payoff vector is characterized by the properties minimal rights first and lower bound of degree half. Well-known examples are the Talmud rule, the adjusted proportional rule and the random arrival rule. Then our focus turns to more specific claim games, i.e. games that use the constrained equal awards rule, the Talmud rule, or the constrained equal losses rule as a sharing rule. Also a variation on the claim game is considered by allowing for arbitrary instead of integer claims.
\end{abstract}

Journal of Economic Literature Classification Nos. C72, D74

Keywords Claim games, estate division problem, bankruptcy problem, Talmud rule, adjusted proportional rule, random arrival rule.

\section{Introduction}

The estate division problem, also known as bankruptcy problem or rationing problem, concerns the issue of dividing an estate among a group of claimants who have entitlements to the estate, when the sum of these entitlements exceeds the size of the estate. A seminal paper on this problem is O'Neill (1982). Subsequently, most research has focused on comparing different solution rules by their properties. For an overview of this normative, axiomatic approach, see Thomson (2003).

The estate division problem can also be approached strategically, i.e., by a non-cooperative game. O'Neill (1982) already formulates a non-cooperative game, associated with an estate division problem, in which players can use their entitlements to claim specific parts of the estate. More precisely, think of an estate with size $E$ as an interval $[0, E]$. Each player can partition this interval into finitely many subintervals and on each of those subintervals put a claim such that the total amount claimed is equal to his entitlement. Then every subinterval is equally divided among those players claiming the subinterval. O'Neill (1982) considers the Nash equilibria of this game. Atlamaz et al. (2011) extend this game by allowing for multiple claims on every subinterval

*Department of Quantitative Economics, Maastricht University, P.O. Box 616, 6200 MD Maastricht, The Netherlands. Email: m.schroeder@maastrichtuniversity.nl, h.peters@maastrichtuniversity.nl, d.vermeulen@maastrichtuniversity.nl 
and dividing every subinterval according to the proportional rule with respect to the claims. We generalize their game by allowing for other possible sharing rules to divide the subintervals.

In by far the larger part of the paper we focus on restricted estate division problems, which means that individual entitlements do not exceed the size of the estate. For the case in which claims are integer-valued, our first result is of an axiomatic nature and applies to problems for which the estate is larger than half of the total entitlements. Under four fairly general axioms: efficiency, claims boundedness, equal treatment of two claims and responsiveness; the set of equilibrium profiles and corresponding payoffs is characterized. We show that a rule always results in an equilibrium payoff vector if and only if the rule satisfies the properties minimal rights first and lower bound of degree half. Three well-known rules that satisfy both properties are the Talmud rule, the adjusted proportional rule and the random arrival rule. This result provides a non-cooperative support to use either of these rules for these kind of problems.

We then direct our attention to claim games that use the constrained equal awards rule, the Talmud rule, or the constrained equal losses rule as a sharing rule. Actually, we consider sharing rules derived from a particular family of rules, the TAL-family, which has been introduced by Moreno-Ternero and Villar (2006) and includes the above three rules. For a characterization result for this family as a whole, we refer to Moreno-Ternero (2011). We characterize all Nash equilibria of the associated claim games and the corresponding payoffs. These results indicate that there is not one general characterization for claims problems with an estate smaller than half of the total entitlements - in contrast to problems with an estate larger than half of the total entitlements.

We also investigate what happens if we relax the assumption of placing integer-valued claims and allow for arbitrary claim heights. Unlike for the proportional case (Atlamaz et al., 2011), the claims profile in which every player has a uniform claim over the estate, is usually not the unique equilibrium claims profile.

Although we use the estate division terminology, our model has applications other than the division of a heritage or the leftovers of a bankrupt firm. For instance, think of the interval $[0, E]$ as representing a continuum of uniformly distributed consumers (cf. Hotelling, 1929), and of the claimants as firms who provide services to the consumers, with total services equal to the entitlements. Every claim can be thought of as an investment in a particular consumer segment. Since we allow for multiple claims, this interpretation allows for competitive investments by different firms in the same consumer segment. As we can choose the sharing rule, it is possible to allow for different forms of competition among the firms. Aside from proportional division of the consumers, one could think of a form of competition in which the firms that invest maximally in a given segment, equally share the consumers in that segment - this is achieved by using the constrained equal losses rule in our model. Or one could imagine a competition in which all investing firms equally share a segment - achieved by using the constrained equal awards rule.

In other related applications the shares induced by the claims may be interpreted as probabilities of winning in, for instance, political elections (cf. Merolla et al., 2005) or auctions (cf. Cramton et al., 2003). Note also the connection to Colonel Blotto games (Borel, 1921).

It is also possible (but postponed to future work) to extend to non-homogeneous preferences over the estate, like in Pálvölgyi et al. (2013). This extension has several additional applications like, for example, land division problems (Berliant et al., 1992). 
The organization of the paper is as follows. In Section 2, we explain the basic model and introduce the relevant sharing rules. In Section 3, we analyze integer claim games for restricted problems with an estate larger than half of the total entitlements. Section 4 also considers integer claim games, but now for more particular sharing rules. In Section 5, players are allowed to place arbitrary claims. Section 6 analyzes the relation between restricted and unrestricted problems. In Section 7 we conclude.

\section{The model}

The set of players is $N=\{1, \ldots, n\}$, where $n \geq 2$. An estate division problem is a pair $(E, c)$, where $E \in \mathbb{R}, E>0$, is the estate and $c=\left(c_{1}, \ldots, c_{n}\right) \in \mathbb{R}^{N}$ with $c_{i}>0$ for all $i \in N$ and $\sum_{i \in N} c_{i} \geq E$, is the vector of entitlements. Let $\mathcal{C}^{N}$ be the class of all problems and let $\mathcal{C}_{0}^{N}$ be the class of all problems with $\sum_{i \in N} c_{i} / 2 \leq E$. A payoff vector for $(E, c) \in \mathcal{C}^{N}$ is a vector $x=\left(x_{1}, \ldots, x_{n}\right) \in \mathbb{R}_{+}^{N}$ with $\sum_{i \in N} x_{i} \leq E$, where $x_{i}$ is the payoff to player $i$, and $\mathbb{R}_{+}:=[0, \infty)$.

The purpose of this paper is to find payoff vectors for estate division problems as equilibrium outcomes of a suitable non-cooperative game. To this end, we first define a sharing rule to be a function $f$ that assigns to every $b \in \mathbb{R}_{+}^{N}$ a vector $f(b) \in[0,1]^{N}$ such that $\sum_{i \in N} f_{i}(b) \leq 1$. Given a sharing rule $f$ we associate with an estate division problem $(E, c) \in \mathcal{C}^{N}$ a claim game, denoted by $(E, c, f)$. First, a strategy of player $i \in N$ in this claim game consist of a finite division of the interval $[0, E]$ into subintervals and on each subinterval a non-negative number of claims, such that the total amount claimed is equal to $c_{i}$. It will be without loss of generality to assume that the strategies of all players have the same division of $[0, E]$ in common, since otherwise we can consider the common refinement of the player divisions instead. The following definition therefore introduces so-called claims profiles, after which we can complete the definition of the game $(E, c, f)$.

Definition 2.1. A claims profile for $(E, c) \in \mathcal{C}^{N}$ is a triple $(y, \beta, m)$, where

(i) $m \in \mathbb{N}$,

(ii) $y=\left(y_{0}, \ldots, y_{m}\right) \in \mathbb{R}^{m+1}$ with $0=y_{0}<y_{1} \ldots<y_{m-1}<y_{m}=E$,

(iii) $\beta=\left(\beta_{1}, \ldots, \beta_{n}\right)$ with $\beta_{i}:\{1, \ldots, m\} \rightarrow \mathbb{R}_{+}$such that

$$
\sum_{t=1}^{m} \beta_{i}(t) \cdot\left(y_{t}-y_{t-1}\right)=c_{i} \text { for all } i \in N \text {. }
$$

We refer to the interval $\left(y_{t-1}, y_{t}\right)$ as interval $t$. We write $\beta(t)=\left(\beta_{i}(t)\right)_{i \in N}$, where $\beta_{i}(t)$ is interpreted as the amount that player $i$ claims on interval $t$; and we write $M=\{1, \ldots, m\}$. We now use the sharing rule $f$ to distribute every interval $t$ among the claimants of the interval. Specifically, $f_{i}(\beta(t))$ is the share of player $i$ of interval $t$, and player $i$ 's payoff is determined by the payoff function $u_{i}^{f}:(y, \beta, m) \mapsto u_{i}^{f}(y, \beta, m) \in \mathbb{R}$ defined by

$$
u_{i}^{f}(y, \beta, m)=\sum_{t \in M} f_{i}(\beta(t)) \cdot\left(y_{t}-y_{t-1}\right)
$$

for every claims profile $(y, \beta, m)$. We write $u^{f}=\left(u_{1}^{f}, \ldots, u_{n}^{f}\right)$. This concludes the definition of the game $(E, c, f)$. 
Atlamaz et al. (2011) analyze this game with the proportional rule as the sharing rule. We generalize their results by considering different sharing rules. We first introduce four properties that a sharing rule may have.

The first requirement states that the interval is distributed if the total claim on it is at least 1.

Efficiency. For each $b \in \mathbb{R}_{+}^{N}$, if $\sum_{i \in N} b_{i} \geq 1$ then $\sum_{i \in N} f_{i}(b)=1$.

The second requirement is that each player receives at most his claim.

Claims boundedness. For each $b \in \mathbb{R}_{+}^{N}$ and each $i \in N, f_{i}(b) \leq b_{i}$.

The next property says that two players obtain equal shares if they have a claim of 1 and are the only two claimants.

Equal treatment of two claims. For each $b \in \mathbb{R}_{+}^{N}$, if $b_{i}=b_{j}=1$ for $i, j \in N$ and $b_{k}=0$ for all $k \in N$, with $k \neq i, j$, then $f_{i}(b)=f_{j}(b)$.

The last property states that for any number of claims of the other players, there is an amount of claims such that the own share is positive.

Responsiveness. For each $b^{-i} \in \mathbb{R}_{+}^{N \backslash\{i\}}$ and each $i \in N$, there is some $b_{i} \in \mathbb{R}_{+}$such that $f_{i}(b)>0 .^{1}$

Let us now define some particular sharing rules derived from the TAL-family, to which the constrained equal awards rule, the constrained equal losses rule and the Talmud rule belong. For the original definitions of these rules see Remark 2.7 .

We first describe the constrained equal awards rule $f^{C E A}$ (as a sharing rule).

Definition 2.2. For each $b \in \mathbb{R}_{+}^{N}$ and each $i \in N$,

$$
f_{i}^{C E A}(b)= \begin{cases}b_{i} & \text { if } \sum_{j \in N} b_{j}<1, \\ \min \left\{b_{i}, \lambda\right\} & \text { if } \sum_{j \in N} b_{j} \geq 1,\end{cases}
$$

where $\lambda$ is the unique solution of the equation $\sum_{j \in N} \min \left\{b_{j}, \lambda\right\}=1$.

The constrained equal awards rule assigns equal shares to all claimants subject to no one receiving more than his claim. Note that $f^{C E A}(0, \ldots, 0)=(0, \ldots, 0)$. When used in a claim game this implies that if a part (an interval) of the estate is not claimed, then it is not distributed. We write $u^{C E A}$ instead of $u^{f^{C E A}}$.

The constrained equal losses rule $f^{C E L}$ (as a sharing rule) is defined as follows.

Definition 2.3. For each $b \in \mathbb{R}_{+}^{N}$ and each $i \in N$,

$$
f_{i}^{C E L}(b)= \begin{cases}b_{i} & \text { if } \sum_{j \in N} b_{j}<1 \\ \max \left\{0, b_{i}-\mu\right\} & \text { if } \sum_{j \in N} b_{j} \geq 1\end{cases}
$$

where $\mu$ is the unique solution of the equation $\sum_{j \in N} \max \left\{0, b_{j}-\mu\right\}=1$.

\footnotetext{
${ }^{1}$ Define $b^{-i}$ as the vector $\left(b_{j}\right)_{j \neq i}$ and $b$ as the vector $\left(b^{-i}, b_{i}\right)$.
} 
The constrained equal losses rule focuses on the loss each claimant incurs. The rule divides these losses equally among all claimants subject to no one receiving a negative amount. Again, in the claim game, if a part of the estate is not claimed, then it is not distributed. We write $u^{C E L}$ instead of $u^{f^{C E L}}$.

The sharing rules derived from the TAL-family $f^{\theta}$, identified by a single parameter $\theta \in[0,1]$, are defined as follows.

Definition 2.4. Let $\theta \in[0,1]$. For each $b \in \mathbb{R}_{+}^{N}$ and each $i \in N$,

$$
f_{i}^{\theta}(b)= \begin{cases}b_{i} & \text { if } \sum_{j \in N} b_{j}<1 \\ \max \left\{\theta b_{i}, b_{i}-\mu\right\} & \text { if } \sum_{j \in N} b_{j} \geq 1 \text { and } \theta \sum_{j \in N} b_{j}<1 \\ \min \left\{\theta b_{i}, \lambda\right\} & \text { if } \theta \sum_{j \in N} b_{j} \geq 1\end{cases}
$$

where $\mu$ is the unique solution of the equation $\sum_{j \in N} \max \left\{\theta b_{j}, b_{j}-\mu\right\}=1$ and $\lambda$ is the unique solution of the equation $\sum_{j \in N} \min \left\{\theta b_{j}, \lambda\right\}=1$.

Every rule from the TAL-family combines the principles of the constrained equal awards rule and the constrained equal losses rule. Namely, if $\theta$ times the aggregate claim is at least one, then no one receives more than a fraction of $\theta$ of his claim. In this case, the constrained equal awards rule is applied with $\theta b$ as claims. If $\theta$ times the aggregate claim is smaller than one, everyone receives at least a $\theta$-fraction of his claim and the remainder is divided using the constrained equal losses rule with $(1-\theta) b$ as claims. This family of rules generalizes the Talmud rule, for which this switch happens exactly halfway, so for $\theta=\frac{1}{2}$. For every $\theta \in[0,1]$, we write $u^{\theta}$ instead of $u^{f^{\theta}}$. Note that $f^{1}=f^{C E A}$ and $f^{0}=f^{C E L}$. We refer to $f^{\frac{1}{2}}$ as the Talmud sharing rule, and denote the corresponding payoff function by $u^{T}$.

Remark. All sharing rules derived from the TAL-family satisfy efficiency, claims boundedness, equal treatment of two claims and responsiveness.

For completeness, we define the proportional rule $f^{P}$ (as a sharing rule), used in Atlamaz et al. (2011).

Definition 2.5. For each $b \in \mathbb{R}_{+}^{N}$ and each $i \in N$,

$$
f_{i}^{P}(b)= \begin{cases}0 & \text { if } b_{i}=0 \\ \frac{b_{i}}{\sum_{j \in N} b_{j}} & \text { if } b_{i}>0\end{cases}
$$

We denote the corresponding vector of payoff functions by $u^{P}$.

Remark. The proportional sharing rule satisfies efficiency, equal treatment of two claims and responsiveness. In general, this rule need not satisfy claims boundedness. However, if we assume integer-valued claims then this condition is satisfied.

Our purpose is to analyze Nash equilibrium outcomes of the claim game under different sharing rules. The following definition is standard.

Definition 2.6. A claims profile $(y, \beta, m)$ is a Nash equilibrium profile (NEP) in $\left(E, c, u^{f}\right)$ if each player maximizes his own payoff, given his opponents' claims. 
Hence, in an NEP, no player can increase his payoff by unilaterally reshuffling his claims. Let the marginal gain or loss be defined as the gain or loss per unit interval from increasing or decreasing one's claim on that interval with a smallest claim unit, which is 1 in the case that only integer claims are allowed, and infinitesimal otherwise. Then, in an NEP, the marginal loss of decreasing one's claim on some interval should be at least as large as the marginal gain of increasing one's claim on some other interval. For proportional sharing this condition is also sufficient for a claim profile to be an NEP (Atlamaz et al., 2011), but this does not hold in general. Suppose, as an example, that claims are restricted to be integer-valued - a case that we will study extensively in Section 3 - and that the constrained equal losses rule is used as the sharing rule; and suppose that $\beta_{i}(t)=2$ for some player $i$ and interval $t$, whereas $\beta_{j}(t)=0$ for all $j \neq i$. If player $j \neq i$ places a claim of size 1 on $t$ his gain on $t$ is zero, hence his marginal gain is zero. If, however, $j$ places a claim of size 2 on $t$ his gain on $t$ is $\frac{1}{2}$ and, thus, his average gain is $\frac{1}{4}$. Marginal and average gains and losses for claim games with integer claims are defined formally in Section 3, where we present a characterization of NEPs in terms of average gains and losses.

We are interested in the payoffs associated with NEPs with respect to different sharing rules. For every claim game $(E, c, f)$ we denote the set of equilibrium payoffs by

$$
U(E, c, f)=\left\{\left(u_{i}^{f}(y, \beta, m)\right)_{i \in N} \mid(y, \beta, m) \text { is an NEP in }(E, c, f)\right\} .
$$

Once the set of equilibrium payoffs for the particular claim games is characterized, our aim is to find rules yielding a payoff within this set of equilibrium payoffs. Formally, a rule is a function $R$ that associates with every $(E, c) \in \mathcal{C}^{N}$ a vector $R(E, c) \in \mathbb{R}_{+}^{N}$ such that $R_{i}(E, c) \leq c_{i}$ for all $i \in N$ and $\sum_{i \in N} R_{i}(E, c)=E$.

Remark 2.7. The original constrained equal awards rule assigns to player $i$ in an estate division problem $(E, c)$ the amount $\min \left\{c_{i}, \lambda\right\}$, where $\lambda$ solves $\sum_{j \in N} \min \left\{c_{j}, \lambda\right\}=E$. Recall that, by assumption, $\sum_{j \in N} c_{j} \geq E$. The original constrained equal losses rule assigns to player $i$ the amount $\max \left\{0, c_{i}-\mu\right\}$, where $\mu$ solves $\sum_{j \in N} \max \left\{0, c_{j}-\mu\right\}=E$. The original TAL-rule with parameter $\theta \in[0,1]$ assigns to player $i$ the amount $\max \left\{\theta c_{i}, c_{i}-\mu\right\}$ if $\theta \sum_{j \in N} c_{j}<E$, and $\min \left\{\theta c_{i}, \lambda\right\}$ if $\theta \sum_{j \in N} c_{j} \geq E$, where $\mu$ and $\lambda$ are the unique solutions of the equations $\sum_{j \in N} \max \left\{\theta c_{j}, c_{j}-\mu\right\}=E$ and $\sum_{j \in N} \min \left\{\theta c_{j}, \lambda\right\}=E$, respectively. The original proportional rule assigns to player $i$ the amount $\frac{c_{i}}{\sum_{j \in N} c_{j}} E$.

The payoffs assigned by the original rules in Remark 2.7 are also obtained by applying the associated sharing rules to the claims profile in which each player puts a constant claim $\frac{c_{i}}{E}$ on the entire estate. ${ }^{2}$ We call this claims profile, the profile $(y, \beta, 1)$, the uniform claims profile.

Example 2.8. Consider the estate division problem $(E, c)$ with $E=4$ and $c=(4,2,1)$. The payoffs assigned to the players by the (original) constrained equal awards rule, the constrained equal losses rule and the Talmud rule are found by considering the uniform claims profile, that is, $\beta(1)=$ $\left(1, \frac{1}{2}, \frac{1}{4}\right)$. Figure 1 illustrates the three sharing rules. The associated shares are $f^{C E A}(\beta(1))=$ $\left(\frac{3}{8}, \frac{3}{8}, \frac{1}{4}\right), f^{C E L}(\beta(1))=\left(\frac{3}{4}, \frac{1}{4}, 0\right)$ and $f^{T}(\beta(1))=\left(\frac{5}{8}, \frac{1}{4}, \frac{1}{8}\right)$. The corresponding payoffs are $u^{C E A}=$ $\left(1 \frac{1}{2}, 1 \frac{1}{2}, 1\right), u^{C E L}=(3,1,0)$ and $u^{T}=\left(2 \frac{1}{2}, 1, \frac{1}{2}\right)$.

A restricted problem is an estate division problem $(E, c)$ with $c_{i} \leq E$ for all $i \in N$. O'Neill (1982) considers claim games with the proportional sharing rule for restricted problems, in which

\footnotetext{
${ }^{2}$ The crucial property here is homogeneity of a rule (Thomson, 2003).
} 


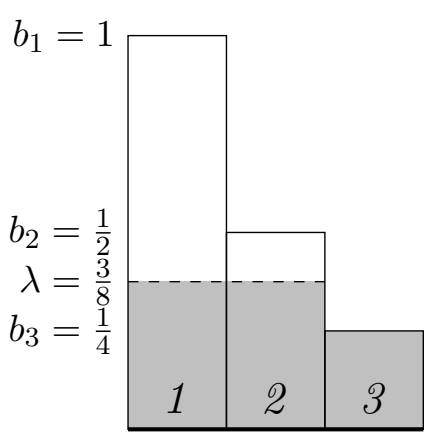

(a)

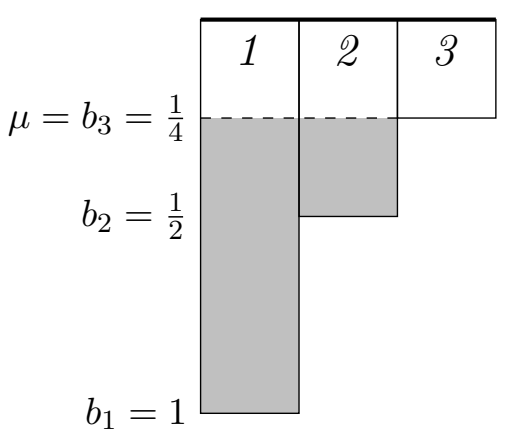

(b)

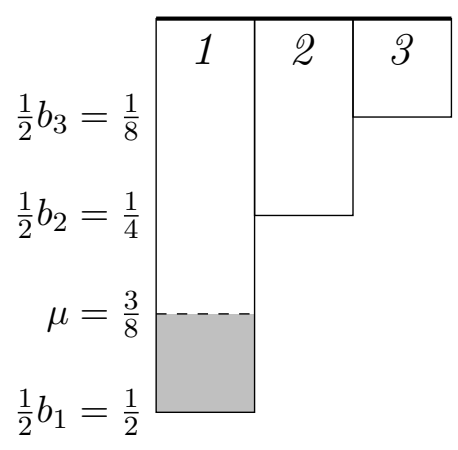

(c)

Figure 1: An illustration of the outcome of different sharing rules for $b=\left(1, \frac{1}{2}, \frac{1}{4}\right)$. The height of every bar represents the claim height and the number in the bar is the name of the player of that claim. (a) The constrained equal awards rule: the height of the shaded area of the bar represents the share of the player. (b) The constrained equal losses rule: the share of each player is represented by the height of the shaded area of the bar. (c) The Talmud rule: every player receives a share of $\frac{1}{2} b_{i}$ plus the height of the shaded area of the bar.

$\beta_{i}(t) \in\{0,1\}$ for each claims profile $(y, \beta, m)$, each $i \in N$, and each $t \in M$. We generalize to multiple and not per se integer claims and different sharing rules. We start with restricted problems and multiple integer claims in the next section.

Notation. We introduce some convenient notation, related to a claims profile $(y, \beta, m)$. For all $t \in M$, we denote $P(t)=\left\{i \in N \mid \beta_{i}(t)>0\right\}, \beta_{\text {min }}(t)=\min _{i \in P(t)} \beta_{i}(t), \beta_{\text {max }}(t)=\max _{i \in P(t)} \beta_{i}(t)$, and $\beta_{N}(t)=\sum_{i \in N} \beta_{i}(t)$.

\section{Restricted problems and integer claims}

In this section we consider restricted problems $(E, c) \in \mathcal{C}^{N}$, i.e., $c_{i} \leq E$ for all $i \in N$, and integer claims in each associated claim game, i.e., $\beta_{i}: M \rightarrow\{0\} \cup \mathbb{N}$ for every claims profile $(y, \beta, m)$ and every $i \in N$. This is the setting also considered in O'Neill (1982), with the difference that $\beta_{i}(t)>1$ is allowed.

Let $f$ be a sharing rule satisfying the minimal requirements efficiency and claims boundedness. We will first define Nash equilibrium profiles (Definition 2.6) in terms of average gains and losses. Let $(y, \beta, m)$ be a claims profile. For $i \in N$ and $t \in M$ with $i \in P(t)$, and $\Delta \in \mathbb{N}$ with $1 \leq \Delta \leq \beta_{i}(t)$, define

$$
A L_{i}(\Delta, t)=\frac{u_{i}^{f}(y, \beta, m)-u_{i}^{f}\left(y, \beta^{\prime}, m\right)}{\left(y_{t}-y_{t-1}\right) \Delta},
$$

where $\left(y, \beta^{\prime}, m\right)$ is a claims profile in the problem $\left(E, c^{\prime}\right)$ such that $\beta^{\prime}$ is equal to $\beta$ except that $\beta_{i}^{\prime}(t)=\beta_{i}(t)-\Delta$, and $c^{\prime}$ is equal to $c$ except that $c_{i}^{\prime}=c_{i}-\left(y_{t}-y_{t-1}\right) \Delta$. Similarly, define

$$
A G_{i}(\Delta, t)=\frac{u_{i}^{f}\left(y, \beta^{\prime \prime}, m\right)-u_{i}^{f}(y, \beta, m)}{\left(y_{t}-y_{t-1}\right) \Delta},
$$


where $\left(y, \beta^{\prime \prime}, m\right)$ is a claims profile in the problem $\left(E, c^{\prime \prime}\right)$ such that $\beta^{\prime \prime}$ is equal to $\beta$ except that $\beta_{i}^{\prime \prime}(t)=\beta_{i}(t)+\Delta$, and $c^{\prime \prime}$ is equal to $c$ except that $c_{i}^{\prime \prime}=c_{i}+\left(y_{t}-y_{t-1}\right) \Delta$. Hence, $A L_{i}(\Delta, t)$ is the average loss (in share) to player $i \in N$ of decreasing his claim on interval $t \in M$ by an amount $\Delta$, and $A G_{i}(\Delta, t)$ is the average gain (in share) of increasing his claim on $t \in M$ by an amount $\Delta$, both measured per unit interval. For $\Delta=1$ these entities are called marginal loss and marginal gain.

The following lemma characterizes Nash equilibrium profiles: a claims profile is an NEP if and only if every interval is positively claimed and for every player the minimum average loss from decreasing his claim on some interval is at least as large as the maximum average gain from increasing his claim on some other interval.

Lemma 3.1. Let $f$ satisfy efficiency and claims boundedness. A claims profile $(y, \beta, m)$ is an NEP in $(E, c, f)$ if and only if $|P(t)| \geq 1$ for all $t \in M$ and for all $i \in N$, we have

$$
\min _{t \in M: i \in P(t)} \min _{\Delta \in\left\{1, \ldots, \beta_{i}(t)\right\}} A L_{i}(\Delta, t) \geq \max _{t \in M} \max _{\Delta \in \mathbb{N}} A G_{i}(\Delta, t) .
$$

Proof. For the only-if part, let claims profile $(y, \beta, m)$ be an NEP. We first show $|P(t)| \geq 1$ for all $t \in M$.

Suppose, contrary to what we want to show, there exists $t \in M$ with $|P(t)|=0$. Since $\sum_{i \in N} c_{i} \geq E$, there exists $t^{\prime} \in M$ with $\beta_{N}\left(t^{\prime}\right) \geq 2$. We consider two cases: $\left|P\left(t^{\prime}\right)\right|=1$ and $\left|P\left(t^{\prime}\right)\right| \geq \overline{2}$.

If $\left|P\left(t^{\prime}\right)\right|=1$, then whenever player $i \in P\left(t^{\prime}\right)$ reduces his claim on $t^{\prime}$ by one, he remains the only claimant and thus by efficiency and claims boundedness $f_{i}(\beta(t))=1$. If player $i$ places this free claim on $t$, then he is the only claimant and gains a positive amount. ${ }^{3}$ This is in contradiction with the NEP assumption.

If $\left|P\left(t^{\prime}\right)\right| \geq 2$, then because a sharing rule distributes at most a total share of one, there is a player $i \in P\left(t^{\prime}\right)$ with $f_{i}\left(\beta\left(t^{\prime}\right)\right)<1$. So $A L_{i}\left(1, t^{\prime}\right)<1$. However, by efficiency and claims boundedness $A G_{i}(1, t)=1$. Hence player $i$ gains a positive amount by reshuffling a claim from $t^{\prime}$ to $t$, which contradicts the NEP assumption.

Next suppose, contrary to (1), there is a player $i$ for which there exist $t, t^{\prime} \in M$ with $i \in P(t)$, $\Delta_{1} \in\left\{1, \ldots, \beta_{i}(t)\right\}$ and $\Delta_{2} \in \mathbb{N}$, such that $A L_{i}\left(\Delta_{1}, t\right)<A G_{i}\left(\Delta_{2}, t^{\prime}\right)$. Taking away $\Delta_{1}$ from (a part of) interval $t$ and placing $\Delta_{2}$ on (a sufficiently small part of) interval $t^{\prime}$ implies an improvement for player $i$, which contradicts that $(y, \beta, m)$ is an NEP.

For the if-part, assume that $|P(t)| \geq 1$ for all $t \in M$ and (1) is satisfied for all $i \in N$. We show that $(y, \beta, m)$ is an NEP.

Consider a claim vector $\bar{\beta}_{i} \neq \beta_{i}$ for player $i \in N$ (potentially resulting in a different partition of $[0, E]$, but in that case we consider the common refinement of both partitions). We argue that the payoff from claim vector $\beta_{i}$ is at least as large as the payoff from $\bar{\beta}_{i}$. The difference in payoff between the two claims profiles arises from intervals on which the claims of player $i$ differ. Let $M_{l}=\left\{t \in M \mid \beta_{i}(t)>\bar{\beta}_{i}(t)\right\}$ and $M_{h}=\left\{t \in M \mid \beta_{i}(t)<\bar{\beta}_{i}(t)\right\}$, respectively denote the intervals with lower and higher claims when going from $\beta_{i}$ to $\bar{\beta}_{i}$. Note that the total difference in claim on intervals from $M_{l}$ is equal to the total difference in claim on intervals $M_{h}$, because player $i$ must

\footnotetext{
${ }^{3}$ In this argument and in many arguments in the sequel, we mean, implicitly, that a player may shift a claim amount from a small enough subinterval of some interval $s$ to a small enough subinterval of some interval $s^{\prime}$.
} 
use his full entitlement. Moreover, the average loss from intervals from $M_{l}$ is at least as high as the average gain from intervals from $M_{h}$, due to (1). Hence the claim vector $\beta_{i}$ is a best response, as it results in a payoff at least as high as the payoff from all other claim vectors.

In the rest of the paper we will often use (sometimes without explicit mentioning) Lemma 3.1 instead of Definition 2.6 when determining Nash equilibria of claim games.

\subsection{Axiomatic approach}

In this subsection, we consider restricted problems $(E, c) \in \mathcal{C}_{0}^{N}$, i.e. $\sum_{i \in N} c_{i} / 2 \leq E$. For this subset of problems, we provide a characterization of all Nash equilibrium profiles if the sharing rule satisfies efficiency, claims boundedness, equal treatment of two claims and responsiveness. ${ }^{4}$

Theorem 3.2. Let $f$ satisfy efficiency, claims boundedness, equal treatment of two claims and responsiveness and let $(y, \beta, m)$ be a claims profile for the restricted problem $(E, c) \in \mathcal{C}_{0}^{N}$. Equivalent are:

(i) $(y, \beta, m)$ is an NEP in $(E, c, f)$.

(ii) $|P(t)| \in\{1,2\}$ and $\beta_{i}(t) \in\{0,1\}$ for all $t \in M$ and $i \in N$.

Proof. We first prove $(i) \Rightarrow(i i)$. Let claims profile $(y, \beta, m)$ be an NEP. By Lemma $3.1,|P(t)| \geq 1$ for all $t \in M$.

Note that responsiveness implies that for all $t \in M$ and all $i \in P(t), f_{i}(\beta(t))>0$. Suppose this claim would not be true, then a player $i \in P(t)$ with $f_{i}(\beta(t))=0$ can improve his payoff. Specifically, player $i$ can reduce his claim on $t$ without any loss. Since $f$ is responsive, there is some $\beta_{i}^{\prime}(t) \in \mathbb{N}$ such that $f_{i}\left(\left(\beta^{-i}(t), \beta_{i}^{\prime}\left(t^{\prime}\right)\right)\right)>0$. So by placing $\beta_{i}^{\prime}(t)$ claims on a sufficiently small subinterval of $t$, player $i$ increases his payoff.

First we show that for all $t \in M$, if $|P(t)|=1$, then $\beta_{i}(t)=1$ for $i \in P(t)$.

Suppose, to the contrary, $|P(t)|=1$ and $\beta_{i}(t)>1$ for $i \in P(t)$. Since player $i$ is the only claimant on $t$, by efficiency and claims boundedness he can reduce his claim on $t$ without any loss. Since we consider restricted problems, there is $t^{\prime} \in M$ with $\beta_{i}\left(t^{\prime}\right)=0$. Since $f$ satisfies responsiveness, there is some $\beta_{i}^{\prime}\left(t^{\prime}\right) \in \mathbb{N}$ such that $f_{i}\left(\left(\beta^{-i}(t), \beta_{i}^{\prime}\left(t^{\prime}\right)\right)\right)>0$. So by placing $\beta_{i}^{\prime}\left(t^{\prime}\right)$ claims on a sufficiently small subinterval of $t^{\prime}$, player $i$ could improve his payoff. This is in contradiction with the assumption that $(y, \beta, m)$ is an NEP.

Second we show that for all $t \in M$, if $|P(t)|=2$, then $\beta_{i}(t)=1$ for each $i \in P(t)$. We distinguish two different cases and use a proof by contradiction for each of these cases.

Suppose $\beta_{i}(t) \geq \beta_{j}(t) \geq 2$ for $i, j \in P(t)$ and $i \neq j$. Then since $f_{i}(\beta(t))>0$ and $f_{j}(\beta(t))>0$, by definition of a sharing rule $f_{i}(\beta(t))<1$ and $f_{j}(\beta(t))<1$. As both players claim $t$ at least twice, $A L_{i}\left(\beta_{i}(t), t\right)<\frac{1}{2}$ and $A L_{j}\left(\beta_{j}(t), t\right)<\frac{1}{2}$.

Since $\sum_{i \in N} c_{i} / 2 \leq E$, there must exist an interval $t^{\prime}$ with $\beta_{N}\left(t^{\prime}\right)=1$. Hence for at least one of these two players (a player without a claim on $t^{\prime}$ ), say player $i, A G_{i}\left(1, t^{\prime}\right)=\frac{1}{2}$ by efficiency, claims

\footnotetext{
${ }^{4}$ Responsiveness requires that for all $i \in N$ and for all $b^{-i} \in \mathbb{R}_{+}^{N \backslash\{i\}}$, there is some $b_{i} \in \mathbb{R}_{+}$such that $f_{i}(b)>0$. Since we consider integer-valued claims, we implicitly assume $b_{i} \in \mathbb{N}$.
} 
boundedness and equal treatment of two claims. This contradicts Lemma 3.1.

Suppose $\beta_{i}(t)>\beta_{j}(t)=1$ for $i, j \in P(t)$. We consider two different possible subcases.

If $0<f_{j}(\beta(t))<\frac{1}{2}<f_{i}(\beta(t))<1$, then $A L_{j}\left(\beta_{j}(t), t\right)<\frac{1}{2}$ and $A L_{i}\left(\beta_{i}(t), t\right)<\frac{1}{2}$ (since $\left.\beta_{i}(t) \geq 2\right)$. Since $\sum_{i \in N} c_{i} / 2 \leq E$, there must exist an interval $t^{\prime}$ with $\beta_{N}\left(t^{\prime}\right)=1$. Hence for at least one of these two players (a player without a claim on $t^{\prime}$ ), say player $i, A G_{i}\left(1, t^{\prime}\right)=\frac{1}{2}$ by efficiency, claims boundedness and equal treatment of two claims. This contradicts Lemma 3.1.

If $0<f_{i}(\beta(t)) \leq \frac{1}{2} \leq f_{j}(\beta(t))<1$, then player $i$ could reduce his claim on $t$ to $\beta_{j}(t)$ such that his share of $t$ equals $\frac{1}{2}$ (by efficiency, claims boundedness and equal treatment of two claims). Either this is an improvement or else he could use his free claim to gain a positive amount of some interval $t^{\prime}$ for which $\beta_{i}\left(t^{\prime}\right)=0$. Since we consider a restricted problem such an interval exists and by responsiveness such a deviation exists.

Finally, we establish a contradiction for any $t \in M$ with $|P(t)| \geq 3$.

Suppose that $|P(t)| \geq 3$. Recall that $f_{i}(\beta(t))>0$ for each $i \in P(t)$. This implies that there are at least two players $i \in P(t)$ with $f_{i}(\beta(t))<\frac{1}{2}$. But then for these two players, $A L_{i}\left(\beta_{i}(t), t\right)<\frac{1}{2}$. Since $\sum_{i \in N} c_{i} / 2 \leq E$, there must exist an interval $t^{\prime}$ with $\beta_{N}\left(t^{\prime}\right)=1$. Hence for at least one of these two players (a player without a claim on $\left.t^{\prime}\right), A G_{i}\left(1, t^{\prime}\right)=\frac{1}{2}$ by efficiency, claims boundedness and equal treatment of two claims. This contradicts Lemma 3.1.

For the converse implication $(i i) \Rightarrow(i)$, assume that $|P(t)| \in\{1,2\}$ and $\beta_{i}(t) \in\{0,1\}$ for all for all $t \in M$ and $i \in N$.

If $|P(t)|=1$, then player $i \in P(t)$ with $f_{i}(\beta(t))=1$ is never able to gain by changing his claim on $t$.

If $|P(t)|=2$, then $A L_{i}(1, t)=\frac{1}{2}$ for $i \in P(t)$. Since for all $t^{\prime} \in M, A G_{i}\left(1, t^{\prime}\right) \leq \frac{1}{2}$ (where $\frac{1}{2}$ can be obtained if $\left|P\left(t^{\prime}\right)\right|=1$ and $i \notin P\left(t^{\prime}\right)$ or if $\left|P\left(t^{\prime}\right)\right|=2$ and $\left.i \in P\left(t^{\prime}\right)\right)$ and $A G_{i}\left(\Delta, t^{\prime}\right) \leq \frac{1}{2}$ for $\Delta \geq 2$ (since a sharing rule distributes at most 1 ), condition (1) of Lemma 3.1 is satisfied. Hence the claims profile constitutes an NEP.

The next result describes every payoff vector in the set of equilibrium payoff vectors obtained in Theorem 3.2.

Corollary 3.3. Let $f$ satisfy efficiency, claims boundedness, equal treatment of two claims and responsiveness and let $(E, c) \in \mathcal{C}_{0}^{N}$ be a restricted problem. The following two statements are equivalent:

(i) $v=\left(v_{i}\right)_{i \in N} \in U(E, c, f)$.

(ii) There exists a vector $r=\left(r_{1}, \ldots, r_{n}\right)$ such that $0 \leq r_{i} \leq \min \left\{c_{i}, \sum_{i \in N} c_{i}-E\right\}$ for every $i \in N$ and $\sum_{i \in N} r_{i}=2 \cdot\left(\sum_{i \in N} c_{i}-E\right)$ with $v_{i}=c_{i}-\frac{1}{2} r_{i}$ for every $i \in N$.

Proof. Suppose $(y, \beta, m)$ is an NEP in $\left(E, c, f^{C E L}\right)$. In view of Theorem 3.2, the length of the part that is claimed by two different players is equal to $\sum_{i \in N} c_{i}-E$ and the length of the part with total claim 1 is equal to $2 E-\sum_{i \in N} c_{i}$. To find the payoff of each player, let $r_{i}$ denote the part of player $i$ 's claim invested in intervals with total claim 2. Then, $c_{i}-r_{i}$ is put on intervals with total claim 1. Clearly, $0 \leq r_{i} \leq c_{i}, r_{i} \leq \sum_{i \in N} c_{i}-E$ and the sum of $r_{i}$ should equal $2 \cdot\left(\sum_{i \in N} c_{i}-E\right)$. These 
conditions imply $c_{i}-r_{i} \leq 2 E-\sum_{i \in N} c_{i}$, since $r_{i}=2 \cdot\left(\sum_{i \in N} c_{i}-E\right)-\sum_{j \in N \backslash i} r_{j} \geq \sum_{i \in N} c_{i}-2 E+c_{i}$. The corresponding payoff for each player $i$ in such a claims profile is equal to

$$
\frac{1}{2} r_{i}+c_{i}-r_{i}=c_{i}-\frac{1}{2} r_{i}
$$

Conversely, suppose there exists a vector $r=\left(r_{1}, \ldots, r_{n}\right)$ satisfying the above conditions. Each such vector $r$ gives rise to an NEP: distribute parts of the entitlement with sizes $r_{i}$ on the interval $\left[0, \sum_{i \in N} c_{i}-E\right]$, such that two players each have a claim of 1 on each part; and distribute the remaining parts of the entitlements $c_{i}-r_{i}$ on the interval $\left[\sum_{i \in N} c_{i}-E, E\right]$, such that each part has total claim 1. To see that this distribution is feasible, note that since $\sum_{i \in N}\left(c_{i}-r_{i}\right)=\sum_{i \in N} c_{i}-2$. $\left(\sum_{i \in N} c_{i}-E\right)=2 E-\sum_{i \in N} c_{i}$ together with $r_{i} \leq c_{i}$, implies that $c_{i}-r_{i} \leq 2 E-\sum_{i \in N} c_{i}$. Hence we found a feasible claims profile that satisfies the conditions of Theorem 3.2, that is, we have an NEP.

Remark 3.4. If there are two players $(n=2)$ then it is easy to see that in an NEP the claims should have minimal overlap. So the only choice for $\left(r_{1}, r_{2}\right)$ is $r_{1}=r_{2}=c_{1}+c_{2}-E$, resulting in the unique equilibrium payoffs $\left(\frac{E+c_{1}-c_{2}}{2}, \frac{E+c_{2}-c_{1}}{2}\right)$. These payoffs coincide with the payoffs assigned by concede-and-divide (Thomson, 2003).

The following example presents an estate division problem for which many well-known rules do not result in an equilibrium payoff vector. ${ }^{5}$ (Atlamaz et al., 2011, give a different example to show that the proportional rule need not be obtained in equilibrium.)

Example 3.5. Consider the restricted problem $(E, c) \in \mathcal{C}_{0}^{N}$ with $E=4$ and $c=(4,2,1)$, cf. Example 2.8. For this problem, $r_{1}=3, r_{2}=2$ and $r_{3}=1$ (see Figure 2). Hence there is a unique equilibrium payoff vector equal to $\left(2 \frac{1}{2}, 1, \frac{1}{2}\right)$.

Every rule from the TAL-family with parameter $\theta \in[0,1]$ assigns a payoff $\theta$ to player 3 . For example, the constrained equal awards rule assigns the payoff vector $\left(1 \frac{1}{2}, 1 \frac{1}{2}, 1\right)$, the Talmud rule assigns the payoff vector $\left(2 \frac{1}{2}, 1, \frac{1}{2}\right)$ and the constrained equal losses rule assigns the payoff vector $(3,1,0)$.

The payoff vector of the proportional rule is equal to $\left(2 \frac{2}{7}, 1 \frac{1}{7}, \frac{4}{7}\right)$, the payoff vector of the adjusted proportional rule is equal to $\left(2 \frac{1}{2}, 1, \frac{1}{2}\right)$, the payoff vector of the random arrival rule is equal to $\left(2 \frac{1}{2}, 1, \frac{1}{2}\right)$, the payoff vector of the reversed Talmud rule is equal to $\left(2 \frac{1}{6}, 1 \frac{1}{6}, \frac{2}{3}\right)$, the payoff vector of the Piniles rule is equal to $\left(2 \frac{1}{6}, 1 \frac{1}{6}, \frac{2}{3}\right)$, the payoff vector of the constrained egalitarian rule is equal to $(2,1,1)$, and the payoff vector of the minimal overlap rule is equal to $\left(2 \frac{5}{6}, \frac{5}{6}, \frac{1}{3}\right)$.

Hence the only rules resulting in an equilibrium payoff are the Talmud rule, the adjusted proportional rule and the random arrival rule.

\footnotetext{
${ }^{5}$ For the definitions of the adjusted proportional rule and the random arrival rule, see the end of this section. For the reversed Talmud rule, see Chun et al. (2001). For the Piniles rule, the constrained egalitarian rule and the minimal overlap rule, see Thomson (2003).
} 


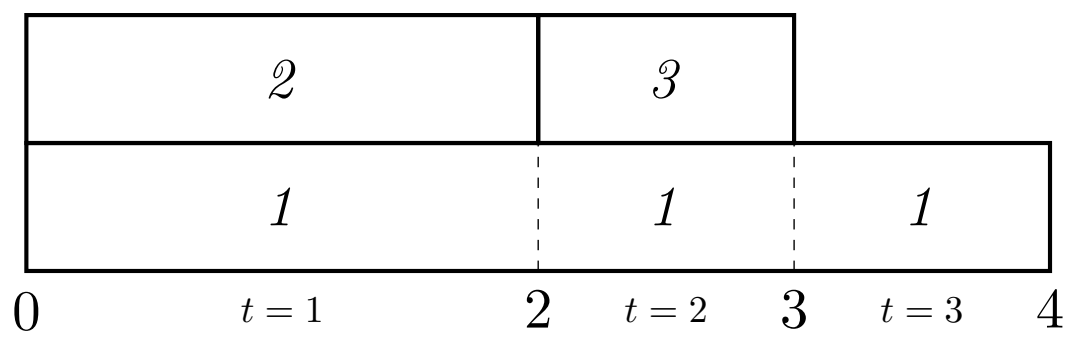

Figure 2: An illustration of a Nash equilibrium claims profile $(y, \beta, m)$ for problem $(E, c)$ with $E=4$ and $c=(4,2,1)$. Each square corresponds to a claim: the number in the square is the name of the player who puts that claim on the interval. Here $r_{1}=3, r_{2}=2$ and $r_{1}=1$.

The above example illustrates that not all rules are supported by an equilibrium argument. In the remainder of this section, we prove that each rule that always leads to an equilibrium vector in claim games with $\sum_{i \in N} c_{i} / 2 \leq E$ must satisfy minimal rights first and lower bound of degree half.

In order to define these properties, we need an additional piece of notation. For $(E, c) \in \mathcal{C}^{N}$, let $m_{i}(E, c)=\max \left\{0, E-\sum_{j \neq i} c_{j}\right\}$ be the minimal right of player $i$ and $m(E, c)=\left(m_{i}(E, c)\right)_{i \in N}$. The first requirement states that the payoff vector is equivalently obtainable (i) directly and (ii) by first assigning the minimal right to each player, adjusting the claims downwards by these amounts and applying the rule to divide the remainder (Curiel et al., 1987).

Minimal rights first. For each $(E, c) \in \mathcal{C}_{0}^{N}, R(E, c)=m(E, c)+R\left(E-\sum_{i \in N} m_{i}(E, c), c-m(E, c)\right)$.

The following property says that each player receives at half his claim.

Lower bound of degree half. For each $(E, c) \in \mathcal{C}_{0}^{N}, R(E, c) \geq \min \{c / 2, E / 2\}$.

It turns out that above two properties describe the equilibrium behavior of a rule.

Theorem 3.6. Let $f$ satisfy efficiency, claims boundedness, equal treatment of two claims and responsiveness. Equivalent are:

(i) $R(E, c) \in U(E, c, f)$ for all restricted $(E, c) \in \mathcal{C}_{0}^{N}$.

(ii) $R$ satisfies minimal rights first and lower bound of degree half.

Proof. We first prove the implication $(i) \Rightarrow(i i)$. Let $(E, c) \in \mathcal{C}_{0}^{N}$ be a restricted problem. By assumption, $R(E, c) \in U(E, c, f)$. By Corollary 3.3, there exists a vector $r=\left(r_{1}, \ldots, r_{n}\right)$ such that $0 \leq r_{i} \leq \min \left\{c_{i}, \sum_{i \in N} c_{i}-E\right\}$ for every $i \in N$ and $\sum_{i \in N} r_{i}=2 \cdot\left(\sum_{i \in N} c_{i}-E\right)$ with $R_{i}(E, c)=c_{i}-\frac{1}{2} r_{i}$ for every $i \in N$. Recall that $r_{i}$ for $i \in N$ specifies the amount of claim placed on an interval with total claims 2 .

We first show that $R$ satisfies minimal rights. Define $K=\left\{i \in N \mid m_{i}(E, c)>0\right\}$. If $i \in K$, then by definition of $m_{i}(E, c), E-\sum_{j \neq i} c_{j}>0$ and thus $c_{i}>\sum_{i \in N} c_{i}-E$. Since $r_{i} \leq \sum_{i \in N} c_{i}-E$, $m_{i}(E, c)$ is placed on an interval with total claim 1 and the minimal right is assigned to player $i$.

Now we show that the revised problem $\left(E-\sum_{i \in N} m_{i}(E, c), c-m(E, c)\right)$ is a restricted problem in $\mathcal{C}_{0}^{N}$ such that $r$ satisfies the conditions of Corollary 3.3 for this problem. To this end, we first prove two claims. 
Claim. $c_{i}-m_{i}(E, c)=\sum_{i \in N} c_{i}-E$ for all $i \in K$.

Proof. By definition of $m_{i}(E, c), c_{i}-m_{i}(E, c)=c_{i}-\left(E-\sum_{j \neq i} c_{j}\right)=\sum_{i \in N} c_{i}-E$.

Claim. $\sum_{i \in N} m_{i}(E, c) \leq 2 E-\sum_{i \in N} c_{i}$.

Proof. Several cases are to be considered.

If $K=\emptyset$, then the claim states $0 \leq 2 E-\sum_{i \in N} c_{i}$ which is true by assumption.

If $|K|=1$, then for $k \in K$ the claim states $E-\sum_{j \in N \backslash k} c_{j} \leq 2 E-\sum_{i \in N} c_{i}$. Straightforward rewriting implies $c_{k} \leq E$, which is true as we consider restricted problems.

If $|K| \geq 2$, then the claim states $|K| \cdot E-|K| \cdot \sum_{j \in N \backslash K} c_{j}-(|K|-1) \cdot \sum_{k \in K} c_{k} \leq 2 E-\sum_{i \in N} c_{i}$. Straightforward rewriting implies

$$
(|K|-2) \cdot E \leq(|K|-2) \cdot \sum_{i \in N} c_{i}+\sum_{j \in N \backslash K} c_{j},
$$

which is true as $\sum_{j \in N \backslash K} c_{j} \geq 0$ and $\sum_{i \in N} c_{i} \geq E$. Hence the Claim is proved.

By the second claim, $E-\sum_{i \in N} m_{i}(E, c) \geq \sum_{i \in N} c_{i}-E$ and thus by the first claim, $0<c_{i}-$ $m_{i}(E, c) \leq E-\sum_{i \in N} m_{i}(E, c)$ for all $i \in N$. Hence the revised problem $\left(E-\sum_{i \in N} m_{i}(E, c), c-m(E, c)\right)$ is a restricted problem. Moreover, the second claim implies $\sum_{i \in N}\left(c_{i}-m_{i}(E, c)\right) / 2 \leq E-\sum_{i \in N} m_{i}(E, c)$. Note the following properties of $r$ :

(i) $r_{i} \geq 0$ for all $i \in N$,

(ii) $r_{i} \leq c_{i}-m_{i}(E, c)$ for all $i \in N$, since if $i \notin K$, then $r_{i} \leq c_{i}$ and if $i \in K$, then $r_{i} \leq$ $\sum_{i \in N} c_{i}-E=c_{i}-m_{i}(E, c)$,

(iii) $r_{i} \leq \sum_{i \in N} c_{i}-E=\sum_{i \in N}\left(c_{i}-m_{i}(E, c)\right)-\left(E-\sum_{i \in N} m_{i}(E, c)\right)$ for all $i \in N$,

(iv) $\sum_{i \in N} r_{i}=2 \cdot\left(\sum_{i \in N} c_{i}-E\right)=2 \cdot\left(\sum_{i \in N}\left(c_{i}-m_{i}(E, c)\right)-\left(E-\sum_{i \in N} m_{i}(E, c)\right)\right)$.

Hence the conditions of Corollary 3.3 are satisfied for the revised problem, which proves that $R(E, c)=m(E, c)+R\left(E-\sum_{i \in N} m_{i}(E, c), c-m(E, c)\right)$.

Next, since $r_{i} \leq c_{i}, R_{i}(E, c)=c_{i}-\frac{1}{2} r_{i} \geq \frac{1}{2} c_{i}$ for all $i \in N$. Hence, $R$ satisfies lower bound of degree half.

We now prove the converse implication $(i i) \Rightarrow(i)$. Let $R$ satisfy minimal rights first and lower bound of degree half and let $(E, c) \in \mathcal{C}_{0}^{N}$ be a restricted problem. Define $r_{i}=2 \cdot\left(c_{i}-R_{i}(E, c)\right)$ for all $i \in N$. We show that $r=\left(r_{1}, \ldots, r_{n}\right)$ satisfies the conditions of Corollary 3.3. Note the following properties of $r$ :

(i) $r_{i} \geq 0$ for all $i \in N$, since $R_{i}(E, c) \leq c_{i}$.

(ii) $r_{i} \leq c_{i}$ for all $i \in N$, since $R_{i}(E, c) \geq \frac{1}{2} c_{i}$ by lower bound of degree half. 
(iii) for all $i \in N$,

$$
\begin{aligned}
r_{i} & =2 \cdot\left(c_{i}-R_{i}(E, c)\right)=2 \cdot\left(c_{i}-m_{i}(E, c)-R_{i}\left(E-\sum_{i \in N} m_{i}(E, c), c-m(E, c)\right)\right) \\
& \leq 2 \cdot\left(c_{i}-m_{i}(E, c)-\frac{1}{2}\left(c_{i}-m_{i}(E, c)\right)\right) \\
& =c_{i}-m_{i}(E, c),
\end{aligned}
$$

where the second equality follows from minimal rights first and the inequality from lower bound of degree half for the revised problem (remember the revised problem is a restricted problem in $\mathcal{C}_{0}^{N}$ ).

If $i \notin K$, then by definition of $m_{i}(E, c), E-\sum_{j \neq i} c_{j} \leq 0$. This implies $r_{i} \leq c_{i} \leq \sum_{i \in N} c_{i}-E$. If $i \in K$, then by the first claim, $r_{i} \leq c_{i}-m_{i}(E, c)=\sum_{i \in N} c_{i}-E$.

(iv) $\sum_{i \in N} r_{i}=\sum_{i \in N} 2 \cdot\left(c_{i}-R_{i}(E, c)\right)=2 \cdot\left(\sum_{i \in N} c_{i}-E\right)$.

Since $R_{i}(E, c)=c_{i}-\frac{1}{2} r_{i}$ for all $i \in N$, Corollary 3.3 implies $R(E, c) \in U(E, c, f)$.

There are three well-known rules that satisfy these two properties: the Talmud rule, the adjusted proportional rule and the random arrival rule. The above result provides a non-cooperative support for these three rules.

Example 3.7. Consider the restricted problem $(E, c) \in \mathcal{C}_{0}^{N}$ with $E=4$ and $c=(3,2,1)$. For this problem, the Talmud rule assigns the payoff vector $\left(2 \frac{1}{4}, 1 \frac{1}{4}, \frac{1}{2}\right)$, the adjusted proportional rule assigns the payoff vector $\left(2 \frac{1}{5}, 1 \frac{1}{5}, \frac{3}{5}\right)$ and the random arrival rule assigns the payoff vector $\left(2 \frac{1}{6}, 1 \frac{1}{6}, \frac{4}{6}\right)$. Hence the three rules can assign a different payoff vector.

Recall that the Talmud rule (Aumann and Maschler, 1985) is the TAL-rule with parameter $\frac{1}{2}$.

Proposition 3.8. The Talmud rule satisfies minimal rights first and lower bound of degree half.

Proof. In Curiel et al. (1987) it is already mentioned that the Talmud rule satisfies minimal rights first. Let $\sum_{i \in N} c_{i} / 2 \leq E$. By definition of the Talmud rule, lower bound of degree half is satisfied.

Next, we consider the adjusted proportional rule (Curiel et al., 1987). Let $t_{i}(E, c)=\min \left\{c_{i}, E\right\}$ be the truncation of player $i$ and $t(E, c)=\left(t_{i}(E, c)\right)_{i \in N}$. The adjusted proportional rule first assigns minimal rights to each player. Second, each claim is revised downwards to the minimum of the remainder and the difference between the initial claim and the minimal right. Third, the remainder is divided proportionally to the revised claims.

Definition 3.9. For each $(E, c) \in \mathcal{C}^{N}$ and each $i \in N$,

$$
A_{i}(E, c)=m_{i}(E, c)+\frac{t_{i}\left(E-\sum_{j \in N} m_{j}(E, c), c-m(E, c)\right)}{\sum_{j \in N} t_{j}\left(E-\sum_{j \in N} m_{j}(E, c), c-m(E, c)\right)} \cdot\left(E-\sum_{j \in N} m_{j}(E, c)\right) .
$$

Proposition 3.10. The adjusted proportional rule satisfies minimal rights first and lower bound of degree half. 
Proof. Curiel et al. (1987) show that the adjusted proportional rule satisfies minimal rights first. We show that the rule satisfies lower bound of degree half for problems with $\sum_{i \in N} c_{i} / 2 \leq E$.

Let $\sum_{i \in N} c_{i} / 2 \leq E$. For each $i \in N$, define $\lambda_{i}=\frac{c_{i}-m_{i}(E, c)}{\sum_{i \in N}\left(c_{i}-m_{i}(E, c)\right)}$. In the proof of Theorem 3.6, it is shown that the revised problem $\left(E-\sum_{i \in N} m_{i}(E, c), c-m(E, c)\right)$ is a restricted problem. Hence the adjusted proportional rule assigns to each player $i \in N, m_{i}(E, c)+\lambda_{i} \cdot\left(E-\sum_{i \in N} m_{i}(E, c)\right)$. Note

$$
\begin{aligned}
m_{i}(E, c)+\lambda_{i} \cdot\left(E-\sum_{i \in N} m_{i}(E, c)\right) & =m_{i}(E, c)+\lambda_{i} \cdot\left(\sum_{i \in N} c_{i}-\sum_{i \in N} m_{i}(E, c)-\left(\sum_{i \in N} c_{i}-E\right)\right) \\
& =c_{i}-\lambda_{i} \cdot\left(\sum_{i \in N} c_{i}-E\right) \\
& \geq c_{i}-\frac{c_{i}-m_{i}(E, c)}{2 \cdot\left(\sum_{i \in N} c_{i}-E\right)} \cdot\left(\sum_{i \in N} c_{i}-E\right)=\frac{1}{2}\left(c_{i}+m_{i}(E, c)\right) \\
& \geq \frac{1}{2} c_{i},
\end{aligned}
$$

where the first inequality follows from the second claim in the proof of Theorem 3.6.

Finally, we consider the random arrival rule (O'Neill, 1982). To define the random arrival rule, imagine players arriving one at a time, and compensate them fully until money runs out. The resulting payoff vector of course depends on the order in which claimants arrive. To remove the unfairness associated with a particular order, take the arithmetic average over all orders of arrival of the payoff vectors calculated in this way. Formally, let $\Pi^{N}$ be the class of bijections from $N$ into itself.

Definition 3.11. For each $(E, c) \in \mathcal{C}^{N}$ and each $i \in N$,

$$
R A_{i}(E, c)=\frac{1}{n !} \sum_{\pi \in \Pi^{N}} \min \left\{c_{i}, \max \left\{0, E-\sum_{j \in N: \pi(j)<\pi(i)} c_{j}\right\}\right\} .
$$

Proposition 3.12. The random arrival rule satisfies minimal rights first and lower bound of degree half.

Proof. In Curiel et al. (1987) it is already mentioned that the random arrival rule satisfies minimal rights first. We show that the random arrival rule satisfies lower bound of degree half for problems with $\sum_{i \in N} c_{i} / 2 \leq E$.

Let $\sum_{i \in N} c_{i} / 2 \leq E$. Let $\pi \in \Pi^{N}$ and let $\pi^{\prime}$ be the permutation in which the order of $\pi$ is reversed. Take $i \in N$, then it is sufficient to show that the average payoff for player $i$ is at least $\frac{1}{2} c_{i}$.

Define $S=\{j \in N \mid \pi(j)<\pi(i)\}$ and $S^{\prime}=\left\{j \in N \mid \pi^{\prime}(j)<\pi^{\prime}(i)\right\}$. Note that $S \cup S^{\prime} \cup\{i\}=N$ and $S \cap S^{\prime}=\emptyset$. We distinguish three cases.

If $S=\emptyset$ or if $|S| \geq 1$ and $\sum_{i \in S} c_{i} \leq E-c_{i}$, then player $i$ is fully compensated in $\pi$. Hence the average payoff of player $i$ over $\pi$ and $\pi^{\prime}$ is at least $\frac{1}{2} c_{i}$.

If $|S| \geq 1$ and $E-c_{i}<\sum_{i \in S} c_{i}<E$, then player $i$ gets a compensation of $E-\sum_{i \in S} c_{i}$ in $\pi$. Since $\sum_{i \in N} c_{i} \leq 2 E, \sum_{i \in S^{\prime}} c_{i}<E$. So player $i$ gets a compensation of $\min \left\{c_{i}, E-\sum_{i \in S^{\prime}} c_{i}\right\}$ in $\pi^{\prime}$. Since $2 E-\sum_{j \neq i} c_{j} \geq c_{i}$, player $i$ 's average payoff over $\pi$ and $\pi^{\prime}$ is at least $\frac{1}{2} c_{i}$. 
If $|S| \geq 1$ and $\sum_{i \in S} c_{i} \geq E$, then player $i$ gets a compensation of 0 in $\pi$. Since $\sum_{i \in N} c_{i} \leq 2 E$, $\sum_{i \in S^{\prime}} c_{i} \leq E-c_{i}$. So player $i$ is fully compensated in $\pi^{\prime}$. Hence the average payoff of player $i$ over $\pi$ and $\pi^{\prime}$ is $\frac{1}{2} c_{i}$.

Since $\pi$ and $\pi^{\prime}$ are chosen arbitrarily and the random arrival rule takes the arithmetic average over all orders of arrival, this completes the proof.

\section{Sharing rules derived from the TAL-family}

We still consider restricted problems with multiple integer claims. The aim of this section is to study claim games with specific sharing rules. In particular, we restrict out attention to sharing rules derived from the TAL-family.

The following lemma is useful for computing average gains and losses if we use a sharing rule derived from the TAL-family.

Lemma 4.1. Let $\theta \in[0,1]$. Let $(y, \beta, m)$ be a claims profile for the game $\left(E, c, f^{\theta}\right)$. Then for all $i \in N$ and $t \in M$,

$$
\min _{\Delta \in\left\{1, \ldots, \beta_{i}(t)\right\}} A L_{i}(\Delta, t)= \begin{cases}A L_{i}\left(\beta_{i}(t), t\right) & \text { if } \theta \beta_{N}(t)<1 \text { and } i \in P(t) \\ A L_{i}(1, t) & \text { if } \theta \beta_{N}(t) \geq 1 \text { and } i \in P(t)\end{cases}
$$

and

$$
\max _{\Delta \in \mathbb{N}} A G_{i}(\Delta, t)=\left\{\begin{array}{c}
\max \left\{A G_{i}(1, t), A G_{i}\left(\beta_{\max }(t)-\beta_{i}(t), t\right),\right. \\
\left.A G_{i}\left(\beta_{\max }(t)+1-\beta_{i}(t), t\right)\right\} \\
\text { if } \theta \beta_{N}(t)<1 \text { and } i \notin P_{\max }(t) \\
A G_{i}(1, t) \quad \text { otherwise }
\end{array}\right.
$$

Proof. We first prove (2). For intervals $t$ with $\theta \beta_{N}(t)<1, f_{i}^{\theta}\left(\beta_{i}(t)\right)=\theta \beta_{i}(t)$ if $i \notin P_{\max }(t)$. So the marginal loss for player $i \in P(t)$ equals $\theta$ if $i \notin P_{\max }(t)$ and is strictly larger than $\theta$ if $i \in P_{\max }(t)$. Thus, the marginal loss either decreases or remains constant as $i$ decreases his claim on $t$. Hence in all such situations, the minimum average loss is obtained when $i$ reduces his claim to zero, i.e., $\Delta=\beta_{i}(t)$.

On the other hand, if $\theta \beta_{N}(t) \geq 1$ then the marginal loss for player $i \in P(t)$ never decreases as $i$ decreases his claim on $t$ such that $\theta \beta_{N}(t) \geq 1$. Moreover, the marginal loss is at most $\theta$ if $\theta \beta_{N}(t) \geq 1$ and at least $\theta$ if $\theta \beta_{N}(t)<1$. These two observations combined imply that the minimum average loss on those intervals is equal to the marginal loss for player $i$, i.e., $\Delta=1$.

We next prove (3). If $\theta \beta_{N}(t)<1$ and $i \notin P_{\max }(t)$ then the maximum average gain on the interval is either the marginal gain (in case $\theta\left(\beta_{N}(t)+\beta_{\max }(t)-\beta_{i}(t)\right) \geq 1$ ), or the average gain of increasing the claim to $\beta_{\max }(t)$ (such that $i$ shares the remainder of $1-\theta\left(\beta_{N}(t)+\beta_{\max }(t)-\beta_{i}(t)\right.$ ) with the other players $j \in P_{\max }(t)$ ), or the average gain of increasing his claim to $\beta_{\max }(t)+1$ (such that $i$ is the only player with the largest claim and thus has no incentive to increase his claim any further).

In all other situations, where either $i \in P_{\max }(t)$ (no incentive to add more than 1 to his claim) or $\theta \beta_{N}(t) \geq 1$ (marginal gain can only decrease if $i$ increases his claim), the maximum average gain is equal to the marginal gain of player $i$.

Remember every rule in the TAL-family is identified by a single parameter $\theta$. In the ensuing subsections we consider the sharing rules $f^{\theta}$ for $\theta \in\left[\frac{1}{2}, 1\right]$ (among which the Talmud and constrained equal awards rule), $\theta=0$ (constrained equal losses rule), and $\theta \in\left(0, \frac{1}{2}\right)$. 


\subsection{Constrained equal awards and Talmud}

Let $\theta \in\left[\frac{1}{2}, 1\right]$. In this subsection we analyze the game $\left(E, c, f^{\theta}\right)$. Recall that among these sharing rules, we have the Talmud sharing rule and the constrained equal awards sharing rule.

Let $(y, \beta, m)$ be a claims profile. Note that if $|P(t)|=1$, then $f_{i}^{\theta}(\beta(t))=1$ for $i \in P(t)$. And if $|P(t)| \geq 2$ for $t \in M$, then the constrained equal awards rule with $\theta \beta(t)$ as entitlements is used to determine the shares. As $\theta \geq \frac{1}{2}$, this implies that the interval is equally divided among the claimants. Thus, for each $i \in N$ and each $t \in M$ :

$$
f_{i}^{\theta}(\beta(t))= \begin{cases}\frac{1}{|P(t)|} & \text { if } i \in P(t) \\ 0 & \text { otherwise. }\end{cases}
$$

In words, each positively claimed interval is equally divided among those who put a positive claim on it. Therefore, the following lemma, which states that in an NEP each interval is claimed at most once by a player, does not come as a surprise.

Lemma 4.2. Let $(y, \beta, m)$ be an NEP. Then $\beta_{i}(t) \in\{0,1\}$ for every $i \in N$ and $t \in M$.

Proof. Let $(y, \beta, m)$ be an NEP. Suppose, contrary to what we wish to prove, that $\beta_{i}(t)>1$ for some $i \in N$ and some $t \in M$. Since $c_{i} \leq E$, there exists an interval $t^{\prime} \in M$ with $\beta_{i}\left(t^{\prime}\right)=0$. Since $A L_{i}(1, t)=0$ and $A G_{i}\left(1, t^{\prime}\right)>0$, we obtain a contradiction with Lemma 3.1.

Since on each interval $t$ each player has a claim of at most 1 in equilibrium, we end up in the same situation as considered by O'Neill (1982). We obtain the following theorem.

Theorem 4.3. Let $\theta \in\left[\frac{1}{2}, 1\right]$ and let $(y, \beta, m)$ be a claims profile for the restricted problem $(E, c) \in$ $\mathcal{C}^{N}$. Equivalent are:

(i) $(y, \beta, m)$ is an NEP in $\left(E, c, f^{\theta}\right)$.

(ii) $|P(t)| \in\{k, k+1\}$, where $k=\left\lfloor\frac{\sum_{i \in N} c_{i}}{E}\right\rfloor$, and $\beta_{i}(t) \in\{0,1\}$ for all $t \in M$ and $i \in N$.

Proof. Suppose $(y, \beta, m)$ is an NEP in $\left(E, c, f^{\theta}\right)$. Since $\sum_{i \in N} c_{i} \geq E$, every part is claimed positively by at least one player. Lemma 4.2 implies that every player puts at most claim 1 on each interval. Consider interval $t$ with the minimum number of claimants and let $k=|P(t)|$. It is sufficient to show that each interval has either $k$ or $k+1$ claimants. Suppose, contrary to what we wish to prove, that there exists an interval $t^{\prime} \in M$ on which the total claim is at least $k+2$. If player $i$ with $\beta_{i}\left(t^{\prime}\right)=1$ claims (a part of) $t$ instead of $t^{\prime}$, his net gain will be at least $\frac{1}{k+1}-\frac{1}{k+2}=\frac{1}{(k+1)(k+2)}>0$, which is a contradiction.

For the converse implication, suppose there exists a $k \in \mathbb{N}$ such that for all $t \in M,|P(t)| \in$ $\{k, k+1\}$ and $\beta_{i}(t) \in\{0,1\}$ for every $i \in N$. Then for all $t \in M, A L_{i}(1, t) \geq \frac{1}{k+1}$ for $i \in P(t)$, and either $A G_{i}(1, t) \leq \frac{1}{k+1}$ for $i \notin P(t)$ or $A G_{i}(1, t)=0$ if $i \in P(t)$. Hence (1) in Lemma 3.1 is satisfied: this claims profile is an NEP.

We refer to the set of equilibrium payoffs in Theorem 4.3 as $U\left(E, c, f^{C E A}\right)$. So $U\left(E, c, f^{C E A}\right)=$ $U\left(E, c, f^{\theta}\right)$ for all $\theta \in\left[\frac{1}{2}, 1\right]$.

The result that the total claim on each interval is either $k$ or $k+1$, is similar to the result found by Atlamaz et al. (2011) for the proportional case. In that case, however, it is possible that a player 
puts two claims on the same interval, which does not happen in the above equilibria. Hence, the set of NEPs for the constrained equal awards rule is a subset of the set of NEPs for the proportional rule. Moreover, if $\beta_{i}(t) \in\{0,1\}$ for every $i \in N$, then the same payoffs are generated. Thus, we have the following result.

Corollary 4.4. Let $(E, c) \in \mathcal{C}^{N}$ be a restricted problem. Then

$$
U\left(E, c, f^{C E A}\right) \subseteq U\left(E, c, f^{P}\right) .
$$

In order to describe the associated payoff vectors, recall that $k=\left\lfloor\frac{\sum_{i \in N} c_{i}}{E}\right\rfloor$. The length of the part with total claim $k+1$ is equal to $\sum_{i \in N} c_{i}-k E$ and the length of the part that with total claim $k$ is equal to $(k+1) E-\sum_{i \in N} c_{i}$. To find the payoff of each player, let $r_{i}$ denote the part of player $i$ 's claim invested in intervals with total claim $k+1$. Then, $c_{i}-r_{i}$ is put on intervals with total claim $k$. Clearly, $0 \leq r_{i} \leq c_{i}, r_{i} \leq \sum_{i \in N} c_{i}-k E$ and the sum of all $r_{i}$ should equal $(k+1) \cdot\left(\sum_{i \in N} c_{i}-k E\right)$. On the other hand, $c_{i}-r_{i} \leq(k+1) E-\sum_{i \in N} c_{i}$. Summarizing, each NEP corresponds to a vector $\left(r_{1}, \ldots, r_{n}\right)$ such that $\sum_{i \in N} r_{i}=(k+1) \cdot\left(\sum_{i \in N} c_{i}-k E\right)$ and for each $i \in N$,

$$
\max \left\{0, \sum_{i \in N} c_{i}+c_{i}-(k+1) E\right\} \leq r_{i} \leq \min \left\{c_{i}, \sum_{i \in N} c_{i}-k E\right\} .
$$

Conversely, each such vector gives rise to an NEP: distribute parts of the entitlement with sizes $r_{i}, i \in N$, on the interval $\left[0, \sum_{i \in N} c_{i}-k E\right]$ such that each part is claimed by an amount of at most 1 and each part is claimed by exactly $k+1$ players; and distribute the remaining parts of the entitlements $c_{i}-r_{i}$ on the interval $\left[\sum_{i \in N} c_{i}-k E, E\right]$ such that each part is claimed by an amount of at most 1 and each part is claimed by exactly $k$ players. Although there are many NEPs associated with the same vector $\left(r_{1}, \ldots, r_{n}\right)$, the corresponding payoff vector is the same for all of them, namely $v=\left(v_{i}\right)_{i \in N}$, given by

$$
v_{i}=\frac{r_{i}}{k+1}+\frac{c_{i}-r_{i}}{k}=\frac{c_{i}}{k}-\frac{r_{i}}{k(k+1)}
$$

for each $i \in N$. This implies that the set of payoff vectors attainable by NEPs is determined by linear inequalities and, in particular, is a polytope.

Example 4.5. Consider the restricted problem $(E, c)$ with $E=4, n=4$, and $c=(4,3,2,1)$. For this problem, $k=2$ and $r_{1}+r_{2}+r_{3}+r_{4}=6$, where $r_{1}=2,1 \leq r_{2} \leq 2,1 \leq r_{3} \leq 2$ (since $0 \leq r_{3}<1$ contradicts with $\left.r_{1}+r_{2}+r_{3}+r_{4}=6\right)$ and $0 \leq r_{4} \leq 1$. Hence, in an NEP in $\left(E, c, f^{C E A}\right)$ player 1 's payoff is $1 \frac{2}{3}$, player 2 's payoff is in $\left[1 \frac{1}{6}, 1 \frac{1}{3}\right]$, player 3 's payoff is in $\left[\frac{2}{3}, \frac{5}{6}\right]$, and player 4 's payoff is in $\left[\frac{1}{3}, \frac{1}{2}\right]$. An example of such a claims profile is represented in Figure 3. The corresponding equilibrium payoffs are given by $\left(1 \frac{2}{3}, 1 \frac{1}{6}, \frac{5}{6}, \frac{1}{3}\right)$. 


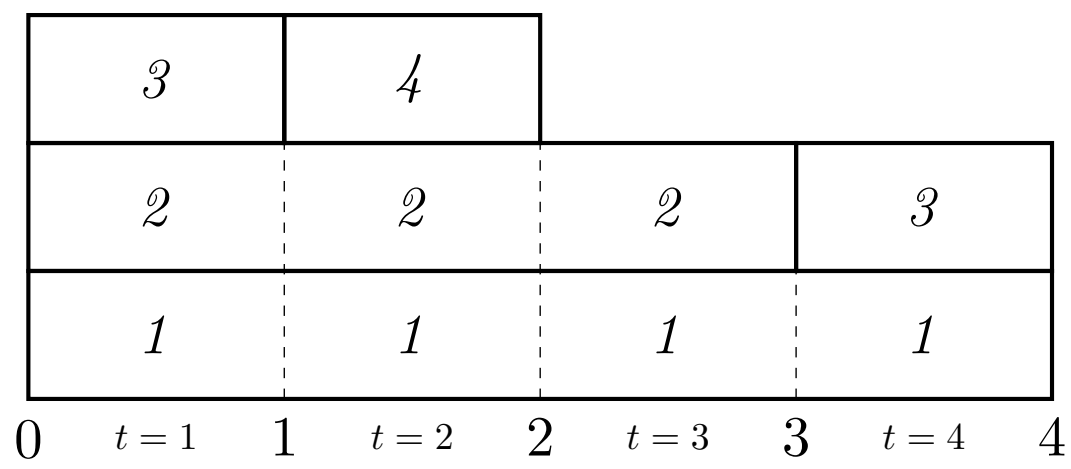

Figure 3: An illustration of a Nash equilibrium claims profile $(y, \beta, m)$ for problem $(E, c)$ with $E=4$ and $c=(4,3,2,1)$. Here $r_{1}=2, r_{2}=2, r_{3}=1$ and $r_{4}=1$.

\subsection{Constrained equal losses}

In this subsection we analyze the game $\left(E, c, f^{C E L}\right)$. For every $t \in M$ we write $P_{\max }(t)=\{i \in N \mid$ $\left.\beta_{i}(t)=\beta_{\max }(t)\right\}$ for the set of players with maximal claim on $t \in M$. Since $\beta_{i}(t) \in \mathbb{N}$ for every $i \in P(t)$, only players $i \in P_{\max }(t)$ obtain a positive share from interval $t$ when the constrained equal losses sharing rule is applied. Observe the resemblance with a first-price auction in which the winners have equal probability to win the object. Formally, for every $i \in N$ and $t \in M$ :

$$
f_{i}^{C E L}(\beta(t))= \begin{cases}\frac{1}{\left|P_{\max }(t)\right|} & \text { if } P(t) \neq \emptyset \text { and } i \in P_{\max }(t), \\ 0 & \text { otherwise. }\end{cases}
$$

For the next two lemmas, let claims profile $(y, \beta, m)$ be an NEP of $\left(E, c, f^{C E L}\right)$. The first lemma implies that every part is claimed by at most two different players.

Lemma 4.6. $\left|P_{\max }(t)\right| \in\{1,2\}$ for every $t \in M$.

Proof. Since $\beta_{N}(t) \geq 1$ for all $t \in M$, we suppose $\left|P_{\max }(t)\right| \geq 3$ for $t \in M$ and derive a contradiction. Consider a player $i$ with $\beta_{i}(t)=\beta_{\max }(t)$. Then $A L_{i}(1, t)=\frac{1}{\left|P_{\max }(t)\right|}$ and $A G_{i}(1, t)=1-\frac{1}{\left|P_{\max }(t)\right|}$. Since $\left|P_{\max }(t)\right| \geq 3$, the marginal loss is smaller than the marginal gain, which contradicts Lemma 3.1 .

The second lemma states that no player puts a claim higher than 1 on an interval.

Lemma 4.7. $\beta_{i}(t) \in\{0,1\}$ for every $i \in N$ and every $t \in M$. Consequently, $\left|P_{\max }(t)\right|=|P(t)|$ for every $t \in M$.

Proof. The proof is by contradiction. Suppose there exists an interval $t \in M$ with $\beta_{\max }(t) \geq 2$. By Lemma 4.6, $\left|P_{\max }(t)\right| \in\{1,2\}$. We derive a contradiction for both cases.

Suppose $\left|P_{\max }(t)\right|=1$ and consider player $i \in P_{\max }(t)$ with $\beta_{i}(t) \geq 2$. If $|P(t)|=1$, then player $i$ can reduce his claim on $t$ without loss and achieve a positive gain by placing this free claim sufficiently often on a part of the estate for which he is not yet the sole winner. If $|P(t)|>1$, then player $j \in P(t) \backslash P_{\max }(t)$ can reduce his claim on $t$ without any loss and put $\beta_{\max }(t)$ claims on a subinterval of $t$ such that he gains a positive amount. 
Suppose that $\left|P_{\max }(t)\right|=2$ with $\beta_{i}(t)=\beta_{j}(t) \geq 2$ for $i, j \in P_{\max }(t)$ and $i \neq j$. Then for $i \in P_{\max }(t), A L_{i}\left(\beta_{i}(t), t\right)=\frac{1}{2 \beta_{i}(t)}$ and $A G_{i}(1, t)=\frac{1}{2}$. Since $\beta_{i}(t) \geq 2$, we obtain a contradiction with Lemma 3.1.

Since $\beta_{i}(t) \in\{0,1\}$ for all $i \in N$ and $t \in M$, the second statement of the lemma follows immediately.

The main result of this subsection is the following theorem, which presents a full characterization of the NEPs.

Theorem 4.8. Let $(y, \beta, m)$ be a claims profile for the restricted problem $(E, c) \in \mathcal{C}^{N}$. Equivalent are:

(i) $(y, \beta, m)$ is an NEP in $\left(E, c, f^{C E L}\right)$.

(ii) $|P(t)| \in\{1,2\}$ and $\beta_{i}(t) \in\{0,1\}$ for all $t \in M$ and $i \in N$.

Proof. Suppose $(y, \beta, m)$ is an NEP in $\left(E, c, u^{C E L}\right)$. Lemmas 4.6 and 4.7 imply that $|P(t)| \in\{1,2\}$ and $\beta_{i}(t) \in\{0,1\}$ for every $i \in N$ and all $t \in M$.

Conversely, assume that $|P(t)| \in\{1,2\}$ and $\beta_{i}(t) \in\{0,1\}$ for all for all $t \in M$ and $i \in N$.

If $|P(t)|=1$, then player $i \in P(t)$ with $f_{i}(\beta(t))=1$ is never able to gain by changing his claim on $t$.

If $|P(t)|=2$, then $A L_{i}(1, t)=\frac{1}{2}$ for $i \in P(t)$. On the other hand, for all $t^{\prime} \in M, A G_{i}\left(1, t^{\prime}\right) \leq \frac{1}{2}$ (where $\frac{1}{2}$ can be obtained if $\left|P\left(t^{\prime}\right)\right|=1$ and $i \notin P\left(t^{\prime}\right)$ or if $\left|P\left(t^{\prime}\right)\right|=2$ and $i \in P\left(t^{\prime}\right)$ ) and $A G_{i}\left(2, t^{\prime}\right)=\frac{1}{2}$ for $i \notin P\left(t^{\prime}\right)$. Hence by Lemma 3.1, the claims profile constitutes an NEP.

Theorem 4.8 shows that the set of NEPs when using the constrained equal losses rule is a subset of the equilibria found for the constrained equal awards rule, which was again a subset of the equilibria found for the proportional rule. Moreover, since $\beta_{i}(t) \in\{0,1\}$ for every $i \in N$, all three rules result in the same payoff vectors. We have the following result.

Corollary 4.9. Let $(E, c) \in \mathcal{C}^{N}$ be a restricted problem. Then

$$
U\left(E, c, f^{C E L}\right) \subseteq U\left(E, c, f^{C E A}\right) \subseteq U\left(E, c, f^{P}\right) .
$$

Another consequence of the Theorem 4.8 is an existence condition for NEP.

Corollary 4.10. An NEP exists in the game $\left(E, c, f^{C E L}\right)$ if and only if $\sum_{i \in N} c_{i} / 2 \leq E$.

Proof. The only-if part follows from Theorem 4.8. For the if-part, note that if $\sum_{i \in N} c_{i} / 2 \leq E$, we can iteratively, from left to right, put the claim of each player on a part of the estate which is not claimed yet and start over again on the left if the total claim on every part of the estate is 1 . Since we consider restricted problems, we will end up with a claim profile satisfying the conditions of Theorem 4.8 . 


\subsection{Remaining sharing rules from the TAL-family}

In this subsection we consider the game $\left(E, c, f^{\theta}\right)$ with $\theta \in\left(0, \frac{1}{2}\right)$.

If $\theta \in\left[\frac{1}{3}, \frac{1}{2}\right)$, then the induced sharing rule is different from the sharing rule with $\theta \in\left[\frac{1}{2}, 1\right]$, but we do obtain the same set of NEPs. (We omit the formal proof, in which the main observation is that if $|P(t)|=2$ for $t \in M$, then $\beta_{i}(t) \leq 2$ for all $i \in N$ and otherwise $\beta_{i}(t) \leq 1$ for all $i \in N$ and all $t \in M$. This is mainly due to the constrained equal awards part of these rules.)

Lemma 4.11. Any rule in the TAL-family with $\theta \in\left[\frac{1}{3}, 1\right]$ results in the same set of NEPs and in the same set of equilibrium payoffs in the game $\left(E, c, f^{\theta}\right)$, equal to $U\left(E, c, f^{C E A}\right)$.

The only claim games that we have not discussed yet, are those associated with problems with $\sum_{i \in N} c_{i} / 2>E$ and a sharing rule $f^{\theta}$ with $\theta \in\left(0, \frac{1}{3}\right)$. We first provide partial answers to the question of existence of NEPs for these kind of games. Later, we provide an example that illustrates that we might need a different characterization for these NEPs (in case they exist).

The next lemma provides a sufficient condition for existence of an NEP. In particular, under this condition there is an $\operatorname{NEP}(y, \beta, m)$ with $\beta_{N}(t) \in\{k, k+1\}$ and $\beta_{i}(t) \in\{0,1\}$ for all $i \in N$.

Lemma 4.12. Let $(E, c) \in \mathcal{C}^{N} \backslash \mathcal{C}_{0}^{N}$ be a restricted problem and let $\theta \geq \frac{k^{2}-k-1}{k^{3}-k}$, where $k=\left\lfloor\frac{\sum_{i \in N} c_{i}}{E}\right\rfloor$. Then there exists an NEP in $\left(E, c, f^{\theta}\right)$.

Proof. Let $(y, \beta, m)$ be a claims profile such that for all $t \in M,|P(t)| \in\{k, k+1\}$ and $\beta_{i}(t) \in\{0,1\}$ for all $i \in N$. Note for all $i \in N, A L_{i}(1, t)=\frac{1}{k}$ for all $t \in M$ with $|P(t)|=k$, and $A L_{i}(1, t)=\frac{1}{k+1}$ for all $t \in M$ with $|P(t)|=k+1$.

In order to prove that condition (1) of Lemma 3.1 is satisfied, we consider two cases: $\frac{k^{2}-k-1}{k^{3}-k} \leq$ $\theta<\frac{1}{k+1}$ and $\theta \geq \frac{1}{k+1}$.

Suppose $\frac{k^{2}-k-1}{k^{3}-k} \leq \theta<\frac{1}{k+1}$. If $t \in M$ is such that $|P(t)|=k$. Then by Lemma 4.1 , it is sufficient to note that for all $i \in P(t), A G_{i}(1, t)=(1-\theta(k-1))-\frac{1}{k}\left(\leq \frac{1}{k+1}\right.$, since $\left.\theta \geq \frac{k^{2}-k-1}{k^{3}-k}\right)$, and for all $i \notin P(t), A G_{i}(1, t)=\frac{1}{k+1}$ and $A G_{i}(2, t)=\frac{1-\theta k}{2}\left(\leq \frac{1}{k+1}\right.$, since $\left.\theta \geq \frac{k^{2}-k-1}{k^{3}-k}\right)$.

If $t \in M$ is such that $|P(t)|=k+1$. Then by Lemma 4.1, it is sufficient to note that for all $i \in P(t), A G_{i}(1, t)=(1-\theta k)-\frac{1}{k+1}\left(\leq \frac{1}{k+1}\right.$, since $\left.\theta \geq \frac{k^{2}-k-1}{k^{3}-k}\right)$, and for all $i \notin P(t), A G_{i}(1, t)=\frac{1}{k+2}$ and either $A G_{i}(2, t)=\frac{1-\theta(k+1)}{2}\left(<\frac{1}{k+1}\right.$, since $\theta \geq \frac{k^{2}-k-1}{k^{3}-k}$ and obtained if $\left.\theta(k+2)<1\right)$ or there is no additional gain for placing a second claim (obtained if $\theta(k+2) \geq 1$ ).

Suppose $\theta \geq \frac{1}{k+1}$. If $t \in M$ is such that $|P(t)|=k$, then by Lemma 4.1, it is sufficient to note that for all $i \in P(t), A G_{i}(1, t) \leq \frac{2}{k+1}-\frac{1}{k}<\frac{1}{k+1}$ (where equality is obtained if $\theta=\frac{1}{k+1}$ ), and for all $i \notin P(t), A G_{i}(1, t)=\frac{1}{k+1}$ and there is no additional gain for placing a second claim.

If $t \in M$ is such that $|P(t)|=k+1$, then by Lemma 4.1, it is sufficient to note that for all $i \in P(t), A G_{i}(1, t)=0$, and for all $i \notin P(t), A G_{i}(1, t)=\frac{1}{k+2}$.

Hence in both cases Lemma 3.1 implies that the claims profile is an NEP.

A consequence of Lemma 4.12 is that if $\theta \geq \frac{5}{24}$ - which is the maximum attained by $\frac{k^{2}-k-1}{k^{3}-k}$, namely for $k=3$ - then there always exists an NEP. 
The following example shows that an NEP need not exist for claim games with $\theta \in\left(0, \frac{1}{6}\right)$.

Example 4.13. Consider the claim game $\left(E, c, f^{\theta}\right)$, with $\theta \in\left(0, \frac{1}{6}\right)$, with $E=1$ and $c=(1,1,1)$. We show that no NEP exists.

To the contrary, suppose that $(y, \beta, m)$ is an NEP. Clearly, $\beta_{N}(t)>0$ for all $t$. We prove the following three claims.

Claim. There is no $t \in M$ with $\beta_{N}(t)=3$.

Proof. Suppose $t \in M$ is such that $\beta_{N}(t)=3$. We show that for all three possible cases there exists a player for which (1) (in Lemma 3.1) does not hold.

If $\beta_{i}(t)=3$ for $i \in P(t)$, then there is $t^{\prime} \in M$ with $\beta_{i}(t)=0$. Since $A L_{i}(1, t)=0$ and $A G_{i}\left(1, t^{\prime}\right)>0$, we obtain a contradiction with Lemma 3.1.

If $\beta_{i}(t)=2$ and $\beta_{j}(t)=1$ for $i, j \in P(t)$ with $i \neq j$, then $A L_{j}(t)=\theta$ and $A G_{j}(t)=\frac{1}{2}-\theta$. Since $0<\theta<\frac{1}{6}$, this contradicts with Lemma 3.1.

If $\beta_{i}(t)=1$ for all $i \in P(t)$, then for $i \in P(t), A L_{i}(1, t)=\frac{1}{3}$ and $A G_{i}(1, t)=(1-2 \theta)-\frac{1}{3}=\frac{2}{3}-2 \theta$. Since $\frac{2}{3}-2 \theta>\frac{1}{3}$ for $0<\theta<\frac{1}{6}$, Lemma 3.1 is violated.

Claim. There is no $t \in M$ with $\beta_{N}(t)=2$.

Proof. Suppose $t \in M$ is such that $\beta_{N}(t)=2$. We show that for both possible cases there exists a player exists for which (1) (in Lemma 3.1) does not hold.

If $\beta_{i}(t)=2$ for $i \in P(t)$, then there is $t^{\prime} \in M$ with $\beta_{i}(t)=0$. Since $A L_{i}(1, t)=0$ and $A G_{i}\left(1, t^{\prime}\right)>0$, we obtain a contradiction with Lemma 3.1 .

If $\beta_{i}(t)=1$ for $i \in P(t)$, then for $i \in P(t), A G_{i}(1, t)=(1-\theta)-\frac{1}{2}=\frac{1}{2}-\theta$ and for $i \notin P(t)$, $A G_{i}(1, t)=\frac{1-2 \theta}{2}=\frac{1}{2}-\theta$. It remains to show that there exists an interval $t^{\prime}$ and a player $i \in N$ for which the average loss on $t^{\prime}$ is strictly less than $\frac{1}{2}-\theta$. Since $\sum_{i \in N} c_{i}=3$ and $\beta_{N}(t)=2$, there exists $t^{\prime} \in M$ with $\beta_{N}\left(t^{\prime}\right) \geq 4$. If there is a player $i \notin P_{\max }\left(t^{\prime}\right)$, then $A L_{i}(1, t) \leq \theta$ and since $\theta<\frac{1}{2}-\theta$ for $0<\theta<\frac{1}{6}$, we are done.

So we may assume $P\left(t^{\prime}\right)=P_{\max }\left(t^{\prime}\right)$. Since $\beta_{N}\left(t^{\prime}\right)=\left|P_{\max }\left(t^{\prime}\right)\right| \cdot \beta_{\max }\left(t^{\prime}\right) \geq 4$, we have $A L_{i}\left(\beta_{\max }\left(t^{\prime}\right), t^{\prime}\right)=\frac{1}{\left|P_{\max }\left(t^{\prime}\right)\right| \cdot \beta_{\max }\left(t^{\prime}\right)} \leq \frac{1}{4}$ for $i \in P_{\max }\left(t^{\prime}\right)$. Since $\frac{1}{4}<\frac{1}{2}-\theta$ for $0<\theta<\frac{1}{6}$, Lemma 3.1 is violated.

Claim. There is no $t \in M$ with $\beta_{N}(t)=1$

Proof. Suppose $t \in M$ is such that $\beta_{N}(t)=1$. We derive a contradiction to (1) in Lemma 3.1.

Consider player $i \in N$ with $\beta_{i}(t)=0$. Since $c_{i}=1$, there exists $t^{\prime} \in M$ with $\beta_{i}\left(t^{\prime}\right) \geq 2$. Note that $\beta_{N}\left(t^{\prime}\right)>\beta_{i}\left(t^{\prime}\right)$, otherwise player $i$ could reduce his claim on $t^{\prime}$ without any loss and place it on $t$, and thus $f_{i}\left(\beta\left(t^{\prime}\right)\right)<1$. But then $A L_{i}\left(2, t^{\prime}\right)<\frac{1}{2}$, while $A G_{i}(1, t)=\frac{1}{2}$. Hence the Claim is proved.

Above three claims imply that $\beta_{N}(t) \geq 4$ for each $t$, which is clearly impossible. Hence, no NEP exists.

From the results in this section, it is clear that there is no general extension for the characterization of NEPs to restricted problems with $\sum_{i \in N} c_{i} / 2>E$. So far, we have seen that the set 
of equilibrium payoffs may differ (compare the games $\left(E, c, f^{C E A}\right)$ and $\left.\left(E, c, f^{P}\right)\right)$ or may even be empty (see the game $\left.\left(E, c, f^{C E A}\right)\right)$. The following example shows that if we consider the game $\left(E, c, f^{\theta}\right)$ with $\theta=\frac{1}{4}$, we yet get a different equilibrium payoff vector.

Example 4.14. Consider the four-player problem $(E, c)$ with $E=2$ and $c=(2,1,2,1)$. Consider the claims profile represented in Figure 4: player 1 claims $[0,1]$ twice and player 2 claims $[0,1]$ once; and player 3 claims $[1,2]$ twice and player 4 claims $[1,2]$ once. This claims profile satisfies the condition in Lemma 3.1 for $\theta=\frac{1}{4}$ and thus is an NEP in $\left(E, c, f^{\frac{1}{4}}\right)$. The corresponding payoff vector is $\left(\frac{3}{4}, \frac{1}{4}, \frac{3}{4}, \frac{1}{4}\right)$. Note that since $k=3$ and $R=0$ (with $k$ and $R$ as in Subsection 4.1), the unique equilibrium payoff vector in the game $\left(E, c, f^{C E A}\right)$ or in the game $\left(E, c, f^{P}\right)$ is $\left(\frac{2}{3}, \frac{1}{3}, \frac{2}{3}, \frac{1}{3}\right)$, hence $\left(\frac{3}{4}, \frac{1}{4}, \frac{3}{4}, \frac{1}{4}\right) \notin U\left(E, c, f^{P}\right)$.

\begin{tabular}{|c|c|}
\hline 2 & 4 \\
\hline 1 & 3 \\
\hline 1 & 3 \\
\hline
\end{tabular}

Figure 4: An illustration of a Nash equilibrium claims profile $(y, \beta, m)$ for $\left(E, c, f^{\theta}\right)$, where $\theta=\frac{1}{4}$, with $E=2$ and $c=(2,1,2,1)$. Notice that both player 1 and 3 claim an interval twice although $R=0$.

The following example shows that the payoff vector from the Talmud rule, or from the adjusted proportional rule, or from the random arrival rule does not need to result in an equilibrium payoff vector in the games $\left(E, c, f^{C E A}\right)$ or $\left(E, c, u^{P}\right)$ if $\sum_{i \in N} c_{i} / 2>E$.

Example 4.15. Consider the restricted problem $(E, c)$ with $n=4, E=4$, and $c=(4,3,2,1)$, cf. Example 4.5. The unique equilibrium payoff of player 1 in $\left(E, c, f^{C E A}\right)$ or in $\left(E, c, f^{P}\right)$ equals $1 \frac{2}{3}$, while $T=\left(1 \frac{1}{4}, 1 \frac{1}{4}, 1, \frac{1}{2}\right), A=\left(1 \frac{3}{5}, 1 \frac{1}{5}, \frac{4}{5}, \frac{2}{5}\right)$ and $R A=\left(1 \frac{7}{12}, 1 \frac{1}{4}, \frac{3}{4}, \frac{5}{12}\right)$.

\section{$5 \quad$ Restricted problems and arbitrary claims}

We still consider restricted problems. For a claims profile $(y, \beta, m)$ in $(E, c)$, we now assume that $\beta_{i}: M \rightarrow \mathbb{R}_{+}$for all $i \in N$. In particular, Lemma 3.1 no longer applies.

Recall that the uniform claims profile is the claims profile in which each player puts a claim of size $\frac{c_{i}}{E}$ on the complete interval $[0, E]$. Also recall that the payoffs assigned by any rule from the TAL-family or by the proportional rule to the estate division problem $(E, c)$ are equal to the payoffs in the claim game associated with that rule under the uniform claims profile.

In the ensuing subsections we consider, respectively, the constrained equal awards, constrained equal losses, and Talmud sharing rules. 


\subsection{Constrained equal awards}

In this subsection we analyze the game $\left(E, c, f^{C E A}\right)$, cf. Definition 2.2. The first lemma shows that the uniform claims profile is an NEP.

Lemma 5.1. The uniform claims profile $(y, \beta, 1)$ is an $N E P$ in $\left(E, c, f^{C E A}\right)$.

Proof. Consider player $i \in N$, then either $u_{i}^{C E A}(y, \beta, 1)=c_{i}$ or $u_{i}^{C E A}(y, \beta, 1)<c_{i}$. In the former case, it is impossible for $i$ to improve. In the latter case, because of the uniform claims profile, $\beta_{i}(t)>\lambda$ for all $t \in M$ (with $\lambda$ as in Definition 2.2). This implies that player $i$ cannot gain by deviating. So $(y, \beta, 1)$ is an NEP.

The following example shows that the uniform claims profile is not necessarily the unique NEP.

Example 5.2. Consider the two-player problem $(E, c)$ with $E=2$ and $c=\left(1 \frac{3}{4}, \frac{3}{4}\right)$. Suppose player 1 puts a claim of $\frac{3}{4}$ on $[0,1]$ and a claim of 1 on $[1,2]$; and player 2 puts a claim of $\frac{1}{2}$ on $[0,1]$ and a claim of $\frac{1}{4}$ on $[1,2]$. This claims profile is an NEP, although it is not the uniform claims profile. Observe that $\lambda=\frac{1}{2}$ on $[0,1]$ and $\lambda=\frac{3}{4}$ on $[1,2]$, so that the associated payoffs are $\left(1 \frac{1}{4}, \frac{3}{4}\right)$. These are the same payoffs the players obtain if the constrained equal awards rule is applied to $(E, c)$.

In this example the equilibrium payoffs are equal to the payoffs assigned by the constrained equal awards rule. The following theorem shows that this is true in general.

Theorem 5.3. All NEPs result in the same payoffs, equal to the payoffs assigned by the constrained equal awards rule.

Proof. Suppose $(y, \beta, m)$ is an NEP in $\left(E, c, f^{C E A}\right)$. Suppose there exists a player $i \in N$ and $t, t^{\prime} \in M$ with $\beta_{i}(t)>\lambda$ and $\beta_{i}\left(t^{\prime}\right)<\lambda$. We derive a contradiction. If $i$ decreases his claim on $t$ to $\lambda$, he will not incur any loss. However, since $\beta_{i}\left(t^{\prime}\right)<\lambda$, increasing his claim on $t^{\prime}$ leads to a positive gain, which contradicts the NEP assumption.

So in an NEP, we have two sets of players: let $J$ denote the set of players with $\beta_{j}(t) \geq \lambda$ for all $t \in M$, where at least one of the inequalities is strict, and let $N \backslash J$ denote the set of players with $\beta_{i}(t) \leq \lambda$ for all $t \in M$.

Note that for all $t \in M, f_{j}^{C E A}(\beta(t))=\lambda$ for all $j \in J$, and $f_{i}^{C E A}(\beta(t))=\beta_{i}(t)$ for all $i \in N \backslash J$. In other words, all players $i \in N \backslash J$ receive exactly their claim. All players $j \in J$ receive the same payoff, which is at least as much as the players $i \in N \backslash J$, but strictly less than their claim. This is precisely the payoff each player obtains from the constrained equal awards rule applied to the estate division problem $(E, c)$.

\subsection{Talmud}

In this subsection we analyze the game $\left(E, c, f^{T}\right)$ : see Definition 2.4 for the definition of $f^{T}=f^{\frac{1}{2}}$. We consider two cases: estate division problems with $\sum_{i \in N} c_{i} / 2>E$ and those with $\sum_{i \in N} c_{i} / 2 \leq$ $E$. We start with the former case.

Theorem 5.4. Let $(E, c) \in \mathcal{C}^{N} \backslash \mathcal{C}_{0}^{N}$ be a restricted problem. Then all NEPs result in the same payoffs, equal to the payoffs assigned by the Talmud rule to $(E, c)$. 
Proof. Suppose $(y, \beta, m)$ is an NEP. Recall that $\beta_{N}(t) \geq 1$ for all $t \in M$. Let $J$ denote the set of players with $\frac{1}{2} \beta_{j}(t)>\lambda$ for some $t \in M$ with $\beta_{N}(t) \geq 2$. Since $\beta_{N}(t)>2$ for some $t \in M$, there exists a player $j \in P(t)$ with $\frac{1}{2} \beta_{j}(t)>\lambda$, which shows that $J \neq \emptyset$. We first prove the statement for $|J|=1$ and afterwards for $|J| \geq 2$.

Suppose that $|J|=1$. Since $j \in J$ can reduce his claim on $t$ to $\lambda$ without any loss (since the constrained equal awards rule is applied on this interval), it should not be possible for him to gain a positive amount from an increase in claim on a different interval $t^{\prime} \neq t$.

If $\beta_{N}\left(t^{\prime}\right) \geq 2$, this implies that $\frac{1}{2} \beta_{j}\left(t^{\prime}\right) \geq \lambda$ for $j \in J$, and that $\frac{1}{2} \beta_{i}\left(t^{\prime}\right) \leq \lambda$ for all $i \in N \backslash\{j\}$ by definition of $J$.

If $\beta_{N}\left(t^{\prime}\right)<2$, this implies by similar arguments that $\frac{1}{2} \beta_{j}\left(t^{\prime}\right) \geq \mu$ for $j \in J$, while for all other players $i \in N \backslash\{j\}$, we have that $\frac{1}{2} \beta_{j}\left(t^{\prime}\right) \leq \mu$, as otherwise $j \in J$ could increase his claim on $t^{\prime}$ with a positive gain.

Hence in equilibrium every player $i \in N \backslash\{j\}$ receives half of his claim, whereas the remainder, which is at least as much as the payoff of every $i \in N \backslash\{j\}$, is for player $j$. These are the same payoffs the players obtain if the Talmud rule is applied to the original estate division problem.

Suppose that $|J| \geq 2$. First, we will argue that $\beta_{N}(t) \geq 2$ for all $t \in M$. By definition of the set $J$, for every player $j \in J$ there exists an interval $t \in M$ with $\beta_{N}(t) \geq 2$ and $\frac{1}{2} \beta_{j}(t)>\lambda$. On this interval $t, j$ can reduce his claim to $\lambda$ without any loss (again because of the constrained equal awards rule). Since we consider an NEP, this means that it must not be possible for $j$ to gain from an increase on any other interval.

Suppose there exists a $t^{\prime} \in M$ with $\beta_{N}\left(t^{\prime}\right)<2$. If there is at most one player $j \in J$ with $\frac{1}{2} \beta_{j}\left(t^{\prime}\right) \geq \mu$, then one of the other players in $J$ gains a positive amount by increasing his claim on $t^{\prime}$. If there are at least two players from $J$ with $\frac{1}{2} \beta_{j}\left(t^{\prime}\right) \geq \mu$, then then either of these players gains a positive amount by increasing his claim on $t^{\prime}$. Since we consider an NEP, these intervals can not exist in equilibrium.

Next we show that $\frac{1}{2} \beta_{j}(t) \geq \lambda$ for all $j \in J$ and for all $t \in M$. Suppose, contrary to what we wish to prove, that $\frac{1}{2} \beta_{j}\left(t^{\prime}\right)<\lambda$ for some $t^{\prime} \in M$. Since $j$ can reduce his claim on some $t \neq t^{\prime}$ without any loss, he can make a positive gain by increasing his claim on $t^{\prime}$. This is a contradiction.

Hence in equilibrium, for all $t \in M, \frac{1}{2} \beta_{j}(t) \geq \lambda$ for all $j \in J$ and $\frac{1}{2} \beta_{i}(t) \leq \lambda$ for all $i \in N \backslash J$ by definition of $J$. This means that all players $i \in N \backslash J$ receive half of their claim on every interval, while all players $j \in J$ receive an equal amount which is at least as much as what the players in $N \backslash J$ receive. Again, these payoffs are equal to the payoffs assigned by the Talmud rule to the original estate division problem.

The following lemma is convenient for finding the equilibrium payoffs in case $\sum_{i \in N} c_{i} / 2 \leq E$. It is the analogue of Lemma 5.12.

Lemma 5.5. Let $(E, c) \in \mathcal{C}_{0}^{N}$ be a restricted problem. For every $N E P$ in $\left(E, c, f^{T}\right)$ there exists a payoff-equivalent $N E P$ such that $|P(t)| \leq 2$ and $\beta_{i}(t)=1$ for all $t \in M$ and $i \in P(t)$.

Proof. Let $(y, \beta, m)$ be an NEP. We will show that we can redistribute the claims on every interval $t \in M$ such that $\left|P\left(t^{\prime}\right)\right| \leq 2$ and $\beta_{i}\left(t^{\prime}\right)=1$ for all $i \in P\left(t^{\prime}\right)$, for every subinterval $t^{\prime}$ of $t$, but without changing any player's total share of the interval $t$. This generates a new finer claim profile which is still an NEP.

Observe that $\beta_{N}(t) \geq 1$ for all $t \in M$, and that $\beta_{i}(t)=1$ for $i \in P(t)$ if $|P(t)|=1$. Thus for this lemma, we only consider intervals with two or more claimants. 
Suppose there is some $t \in M$ with $\beta_{N}(t)>2$. There can only be one player $j$ with $\frac{1}{2} \beta_{j}(t)>\lambda$ (otherwise one of such players can gain by increasing his claim on $t^{\prime} \neq t$ with $\beta_{N}\left(t^{\prime}\right)<2$ ). Because $j$ can reduce his claim to $\lambda$ without any loss and since we consider an NEP, $j$ can increase his claim on any $t^{\prime} \neq t$ without any gain. As $\sum_{i \in N} c_{i} / 2 \leq E$, it is thus possible to redistribute $j$ 's claims such that all $t \in M$ satisfy $\beta_{N}(t) \leq 2$ but without changing the shares of the players.

Thus, w.l.o.g., we can assume that all $t \in M$ satisfy $1 \leq \beta_{N}(t) \leq 2$. For all $t \in M$, divide $P(t)$ into two different groups. Let $J(t)$ denote the set of players $j$ for who $\beta_{j}(t)-\mu>\frac{1}{2} \beta_{j}(t)$, then $P(t) \backslash J(t)$ is the set of players $i$ for who $\beta_{i}(t)-\mu \leq \frac{1}{2} \beta_{i}(t)$. This means that the share of player $j \in J(t)$ is equal to $\beta_{j}(t)-\mu$ and the share of player $i \in P(t) \backslash J(t)$ is equal to $\frac{1}{2} \beta_{i}(t)$, where

$$
\mu=\frac{1}{|J(t)|}\left(\sum_{j \in J(t)} \beta_{j}(t)+\sum_{i \in P(t) \backslash J(t)} \frac{1}{2} \beta_{i}(t)-1\right) \text { if } J(t) \neq \emptyset .
$$

We show that for some $y_{t-1}<\alpha<y_{t}$, we can reshuffle all the claims on $\left(y_{t-1}, y_{t}\right)$ such that on every subinterval of $\left(y_{t-1}, \alpha\right)$ there is a claim of 1 by two players, on every subinterval of $\left(\alpha, y_{t}\right)$ there is a claim of 1 by one player, and all shares of players $i \in P(t)$ remain unchanged. The procedure we use here is similar to the way we describe the payoffs in Corollary 3.3, using the vector $r$, only now applied to the specific interval $t$. Since $\beta_{N}(t)\left(y_{t}-y_{t-1}\right)=2\left(\alpha-y_{t-1}\right)+\left(y_{t}-\alpha\right)$, we have $\alpha=y_{t-1}+\left(\beta_{N}(t)-1\right)\left(y_{t}-y_{t-1}\right)$.

Let $x_{j}=2 \mu$ for all $j \in J(t)$ and $x_{i}=\beta_{i}(t)$ for all $i \in P(t) \backslash J(t)$ denote the part of the claim distributed on $\left(y_{t-1}, \alpha\right)$ such that on every part there is a claim of 1 by two players, and let $\beta_{j}(t)-x_{j}$ for all $j \in J(t)$ be distributed on $\left(\alpha, y_{t}\right)$ such that on every part there is a claim of 1 by one player.

In order to see that we have a feasible redistribution, note the following properties:

(i) $2 \mu \geq 0$.

(ii) $2 \mu<\beta_{j}(t)$ for all $j \in J(t)$, since $\beta_{j}(t)-\mu>\frac{1}{2} \beta_{j}(t)$.

(iii) If $|J(t)| \geq 1$, then $\beta_{i}(t) \leq 2 \mu \leq \beta_{N}(t)-1$ for all $i \in P(t) \backslash J(t)$. Since $\beta_{j}(t) \leq 1$ for $j \in J(t)$ if $|J(t)|=1$ and $(|J(t)|-1) \beta_{N}(t)-\sum_{j \in J(t)} \beta_{j}(t) \geq(|J(t)|-2) \beta_{N}(t) \geq(|J(t)|-2)$ if $|J(t)| \geq 2$.

(iv) $\sum_{i \in P(t)} x_{i}=\sum_{j \in J} 2 \mu+\sum_{i \in P(t) \backslash J} \beta_{i}(t)=2\left(\beta_{N}(t)-1\right)$.

Moreover, the share of player $j \in J(t)$ is

$$
\frac{1}{2} x_{j}+\beta_{j}(t)-x_{j}=\beta_{j}(t)-\mu,
$$

and for player $i \in P(t) \backslash J(t)$ it is

$$
\frac{1}{2} x_{i}=\frac{1}{2} \beta_{i}(t)
$$

If we reshuffle every interval in the above way, we end up with an equilibrium claims profile without changing the shares of the players.

We are now able to describe the payoffs associated with every NEP. If $\sum_{i \in N} c_{i} / 2>E$, then Theorem 5.4 applies, which means that the equilibrium payoffs are equal to the payoffs from the Talmud rule. If $\sum_{i \in N} c_{i} / 2 \leq E$, by Lemma 5.5 all NEP payoffs can be found by only using intervals with either one or two claimants. Hence the set of payoff vectors is equal to the set in Corollary 3.3 . 


\subsection{Constrained equal losses}

In this subsection we analyze the game $\left(E, c, f^{C E L}\right)$, cf. Definition 2.3. The following example shows that the uniform claims profile is not always an NEP.

Example 5.6. Consider the three-player problem $(E, c)$ with $E=4$ and $c=(4,2,1)$. The payoffs in the uniform claims profile are $(3,1,0)$. If player 3 puts claim 1 on $[0,1]$ instead, he receives payoff $\frac{1}{2}$. Thus, the uniform claims profile is not an NEP in $\left(E, c, f^{C E L}\right)$.

The following lemma provides a necessary condition for an NEP in the game $\left(E, c, f^{C E L}\right)$.

Lemma 5.7. Let $(y, \beta, m)$ be an NEP. Then $\beta_{N}(t)-\beta_{\min }(t) \leq 1$ for all $t \in M$.

Proof. Let $t \in M$. The statement trivially holds in case $|P(t)|=1$. For the other cases, we argue by contradiction.

Suppose that $\beta_{N}(t)-\beta_{\min }(t)>1$, which implies that $|P(t)| \geq 2$. We will show that a player $i \in P(t)$ with $\beta_{i}(t)=\beta_{\text {min }}(t)$ can gain by deviating. Observe that in an NEP: $f_{j}(\beta(t))>0$ for all $j \in P(t)$. Otherwise, a player $j$ with $f_{j}(\beta(t))=0$ could put his claim on a sufficiently small subinterval of $t$ in order to gain a positive amount. Let $i$ divide $t$ into two equally large intervals. We will now show that $i$ can transfer an amount of $r$ from his claim on the first half to the second half, such that his share on the first half equals zero but without changing anyone's total share of the interval (including his own share). The remaining claim on the first half can then be used to increase his total share of $t$, since the marginal loss on the first half after the transfer is zero.

Since the losses are equally distributed among all claimants, the decrease in claim on the first half, leads to a decrease in $\mu$ (with $\mu$ as in Definition 2.3) at a constant rate of $\frac{1}{|P(t)|}$. On the second half, the increase in claim increases $\mu$ at a constant rate of $\frac{1}{|P(t)|}$, until some claimant's share drops to zero. We will show that during this procedure all claimants' shares remain positive on the second half, which implies that the total share of every player on $t$ stays the same. To this end, see Figure 5 .

At the point $r=r^{*}=\frac{|P(t)|\left(\beta_{i}(t)-\mu\right)}{|P(t)|-1}$, we see that $\mu_{1}(r)$ - i.e., the new value of $\mu$ on the first half after a transfer of $r$-intersects with the line $\beta_{i}(t)-r$. This means that we are at the point at which the share of player $i$ dropped to zero on the first half. In order to see what happens on the second half, note that

$$
\mu_{2}\left(r^{*}\right)=\frac{1}{|P(t)|} r^{*}+\mu=\frac{\beta_{i}(t)}{|P(t)|-1}+\frac{(|P(t)|-2) \mu}{|P(t)|-1} .
$$

In view of the right-hand side of the expression, we will first treat the case $|P(t)|=2$ separately.

If $|P(t)|=2$ with $\beta_{i}(t)=\beta_{j}(t)>1$ for $i, j \in P(t)$, then $\mu_{2}\left(r^{*}\right)=\beta_{i}(t)=\beta_{j}(t)$ - where $\mu_{2}(r)$ is, analogously, the new value of $\mu$ on the second half. This implies that the share of player $j$ dropped to zero on the second half after the transfer, which means that $i$ has a share of 1 on the second half and $j$ has a share of 1 on the first half. Notice that the remaining claim of $\mu_{1}\left(r^{*}\right)=\beta_{i}(t)-1$ on the first half can be placed on a sufficiently small subinterval of the first half such that $i$ gains a positive amount on this first half as well. This, however, means that $i$ is able to gain by deviating, which is a contradiction.

On the other hand, if $|P(t)|=2$ with $\beta_{i}(t)<\beta_{j}(t)$ for $i, j \in P(t)$ or if $|P(t)| \geq 3$ then $\mu_{2}\left(r^{*}\right)<\beta_{j}(t)$ for all $j \in P(t) \backslash i$. This means that the share of every player $j \in P(t) \backslash i$ remains positive on the second half after the transfer of $i$. This conclusion is obvious for $|P(t)|=2$ with 


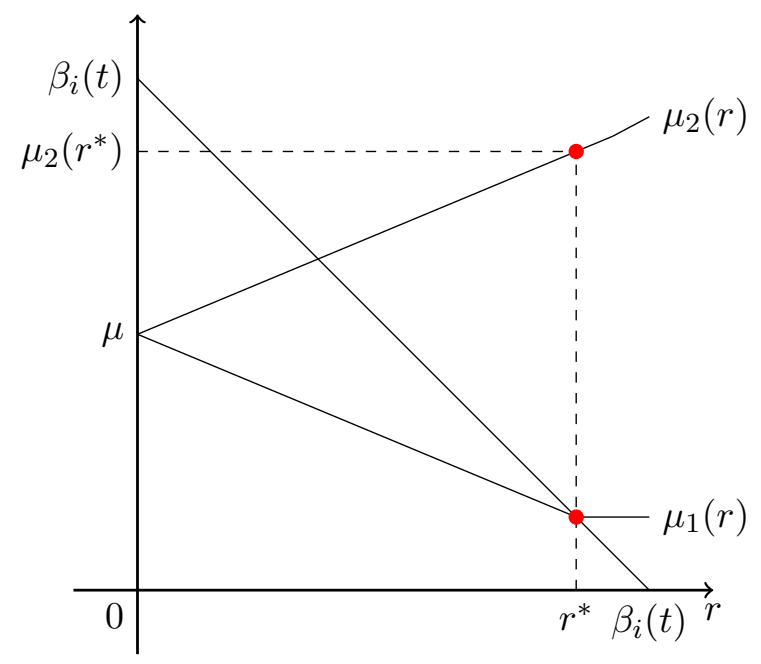

Figure 5: An illustration of the level of $\mu$ on the first and second half of $t$. The value of $r$ represents the amount of claim transferred from the first to the second half of $t$. The line $\mu_{1}(r)$ denotes the level of $\mu$ on the first half of $t$ and the line $\mu_{2}(r)$ denotes the level of $\mu$ on the second half of $t$. The line $\beta_{i}(t)-r$ represents the amount of claim of player $i$ left on the first half of the interval. Notice that the slope of $\mu_{1}(r)$ is $\frac{-1}{|P(t)|}$ until $r^{*}$ and the slope of $\mu_{2}(r)$ is $\frac{1}{|P(t)|}$ until $r^{*}$.

$\beta_{i}(t)<\beta_{j}(t)$, since then $\mu_{2}\left(r^{*}\right)=\beta_{i}(t)<\beta_{j}(t)$. For $|P(t)| \geq 3$, notice that it is sufficient to show that $\mu_{2}\left(r^{*}\right)<\beta_{i}(t)$ (as $\beta_{i}(t) \leq \beta_{j}(t)$ for all $j \in P(t) \backslash i$ ), which is equivalent to showing $\mu<\beta_{i}(t)$. This is true since $f_{j}(\beta(t))>0$ for all $j \in P(t)$, thus in particular for player $j$ himself.

In order to show that $i$ can actually gain by deviating, observe that since $\mu_{1}\left(r^{*}\right)=\frac{\beta_{N}(t)-\beta_{i}(t)-1}{n-1}>$ 0 by assumption, player $i$ could decrease his claim by an additional positive, but sufficiently small, amount of $r$ on the first half without any loss, while having a marginal gain of $1-\frac{1}{|P(t)|}$ on the second half. This contradicts that $(y, \beta, m)$ is an NEP.

As a corollary to the previous lemma, we obtain a necessary and sufficient condition for the uniform claims profile to be an NEP.

Corollary 5.8. The uniform claims profile is an $N E P$ in $\left(E, c, f^{C E L}\right)$ if and only if $\sum_{i \in N} c_{i}-$ $\min _{i \in N} c_{i} \leq E$.

Proof. If the uniform claims profile is an NEP, then Lemma 5.7 implies $\sum_{i \in N} \frac{c_{i}}{E}-\min _{i \in N} \frac{c_{i}}{E} \leq 1$, which is the only-if statement. For the if-part, suppose that $\sum_{i \in N} c_{i}-\min _{i \in N} c_{i} \leq E$ and consider the uniform claims profile. The average gain of any increase on an interval is at most $\frac{n-1}{n}$, as the increased loss is equally divided among all players as long as the shares remain positive. The average loss of a decrease is at least $\frac{n-1}{n}$ by similar arguments. Hence, no player has a profitable deviation.

The following theorem gives a full description of every possible NEP. We restrict our attention to those estate division problems with $\sum_{i \in N} c_{i}>E$. The reason for this is that if $\sum_{i \in N} c_{i}=E$ then in equilibrium every interval $t \in M$ satisfies $\beta_{N}(t)=1$, which means that everyone receives his claim. 
Theorem 5.9. Let $(y, \beta, m)$ be a claims profile for the restricted problem $(E, c) \in \mathcal{C}^{N}$ with $\sum_{i \in N} c_{i}>$ $E$. Then the following statements are equivalent:

(i) $(y, \beta, m)$ is an NEP in $\left(E, c, f^{C E L}\right)$.

(ii) Let $k=\max \{|P(t)| \mid t \in M\}$. Then $k \geq 2$ and the following three conditions are satisfied:

(a) For all $t \in M$, if $|P(t)|<k$, then $\beta_{N}(t)=1$.

(b) For all $t \in M$, if $|P(t)|=k$ and $P(t)=P\left(t^{\prime}\right)$ for all $t^{\prime} \in M$ with $\left|P\left(t^{\prime}\right)\right|=k$, then $1-\beta_{\min }(t) \leq \beta_{N}(t)-\beta_{\min }(t) \leq 1$.

(c) For all $t \in M$, if $|P(t)|=k$ and $P(t) \neq P\left(t^{\prime}\right)$ for some $t^{\prime} \in M$ with $\left|P\left(t^{\prime}\right)\right|=k$, then $\beta_{N}(t)-\beta_{\min }(t)=1$.

Proof. For the implication $(i) \Rightarrow(i i)$, let $(y, \beta, m)$ be an NEP in $\left(E, c, f^{C E L}\right)$ and define $k$ as in $(i i)$. Observe that since a player can not be the sole winner of every interval, $\beta_{N}(t)=1$ if $|P(t)|=1$. This implies that $k \geq 2$ and that we only need to consider intervals $t \in M$ with $|P(t)| \geq 2$. By Lemma 5.7 and since $\beta_{N}(t) \geq 1$ for all $t \in M$, we have that all $t \in M$ with $|P(t)| \geq 2$ satisfy

$$
1-\beta_{\min }(t) \leq \beta_{N}(t)-\beta_{\min }(t) \leq 1 \text {. }
$$

This proves $(b)$.

Lemma 5.7 only considers deviations within a specific interval. The following claim, which is used to prove $(a)$ and $(c)$, considers deviations between two different intervals. For the remainder of this proof, we denote $\beta_{(1)}(t) \geq \beta_{(2)}(t) \geq \ldots \geq \beta_{(|P(t)|)}(t)>0$ for all $t \in M$.

Claim. Let there exists a player $i \in P\left(t^{\prime}\right), i \notin P(t)$, where $t, t^{\prime} \in M$ with $2 \leq\left|P\left(t^{\prime}\right)\right| \leq|P(t)|$ satisfy (4) with $\beta_{N}\left(t^{\prime}\right)>1$. Then $\sum_{i=1}^{\left|P\left(t^{\prime}\right)\right|-1} \beta_{(i)}(t) \geq 1$.

Proof of claim. In an NEP, player $i$ is not able to gain by putting some of his claim of interval $t^{\prime}$ on interval $t$. The average loss of a sufficiently small decrease in claim on $t^{\prime}$ equals $1-\frac{1}{\left|P\left(t^{\prime}\right)\right|}$.

The best player $i$ can do on $t$ is to place a claim such that $\mu=\beta_{\left(\left|P\left(t^{\prime}\right)\right|\right)}(t)$, meaning that at most $\left|P\left(t^{\prime}\right)\right|-1$ other players have a positive share left. Player $i$ cannot do better, since a further increase would lead to a marginal gain of at most $1-\frac{1}{\left|P\left(t^{\prime}\right)\right|}$, whereas placing a lower claim would mean that the opportunity of a marginal gain of at least $\frac{\left|P\left(t^{\prime}\right)\right|}{\left|P\left(t^{\prime}\right)\right|+1}$ will be ignored. More precisely, let player $i$ put a claim of size $\beta_{i}(t)=\left|P\left(t^{\prime}\right)\right| \beta_{\left(\left|P\left(t^{\prime}\right)\right|\right)}(t)-\sum_{j=1}^{\left|P\left(t^{\prime}\right)\right|-1} \beta_{(j)}(t)+1$ on a $\delta$-fraction of $t$, where $0<\delta \leq 1$ is chosen such that the average loss on $t^{\prime}$ does not exceed $\frac{\left|P\left(t^{\prime}\right)\right|-1}{\left|P\left(t^{\prime}\right)\right|}$. His loss in payoff from $t^{\prime}$ is then equal to the total amount of claim needed times the average loss:

$$
\begin{gathered}
\beta_{i}(t) \delta\left(y_{t}-y_{t-1}\right)\left(1-\frac{1}{\left|P\left(t^{\prime}\right)\right|}\right)= \\
\left(\left|P\left(t^{\prime}\right)\right| \beta_{\left(\left|P\left(t^{\prime}\right)\right|\right)}(t)-\sum_{j=1}^{\left|P\left(t^{\prime}\right)\right|-1} \beta_{(j)}(t)+1\right) \delta\left(y_{t}-y_{t-1}\right) \frac{\left|P\left(t^{\prime}\right)\right|-1}{\left|P\left(t^{\prime}\right)\right|} .
\end{gathered}
$$

Since $\mu=\beta_{\left(\left|P\left(t^{\prime}\right)\right|\right)}(t)$, the gain in payoff from $t$ is equal to

$$
\left(\beta_{i}(t)-\mu\right) \delta\left(y_{t}-y_{t-1}\right)=\left(\left(\left|P\left(t^{\prime}\right)\right|-1\right) \beta_{\left(\left|P\left(t^{\prime}\right)\right|\right)}(t)-\sum_{j=1}^{\left|P\left(t^{\prime}\right)\right|-1} \beta_{(j)}(t)+1\right) \delta\left(y_{t}-y_{t-1}\right) .
$$


Since the loss in payoff must be at least as large as the gain in payoff, we get after tedious rewriting:

$$
\sum_{i=1}^{\left|P\left(t^{\prime}\right)\right|-1} \beta_{(i)}(t) \geq 1
$$

This completes the proof of the Claim.

For $(a)$, suppose that $\beta_{N}\left(t^{\prime}\right)>1$ for $t^{\prime} \in M$ with $\left|P\left(t^{\prime}\right)\right|<k$. We will derive a contradiction. Consider an interval $t$ with $|P(t)|=k$. Observe that there cannot be a player $i \in P\left(t^{\prime}\right) \cap P(t)$ (since then his marginal loss on $t^{\prime}$ would be smaller than his marginal gain on $t$ ). So there exists a player $i \in P\left(t^{\prime}\right), i \notin P(t)$. From the claim, $\sum_{j=1}^{\left|P\left(t^{\prime}\right)\right|-1} \beta_{(j)}(t) \geq 1$. This, however, contradicts with (4), since

$$
\beta_{N}(t)-\beta_{\min }(t)=\sum_{j=1}^{k-1} \beta_{(j)}(t)=\sum_{j=1}^{\left|P\left(t^{\prime}\right)\right|-1} \beta_{(j)}(t)+\sum_{j=\left|P\left(t^{\prime}\right)\right|}^{k-1} \beta_{(j)}(t) \geq 1+\sum_{j=\left|P\left(t^{\prime}\right)\right|}^{k-1} \beta_{(j)}(t)>1 .
$$

Hence we have shown $(a)$.

For $(c)$, note that all $t \in M$ with $|P(t)|<k$ satisfy $\beta_{N}(t)=1$. This combined with $\sum_{i \in N} c_{i}>E$, implies that there exists an interval $t^{\prime} \in M$ with $\left|P\left(t^{\prime}\right)\right|=k$ and $\sum_{i \in P\left(t^{\prime}\right)} \beta_{i}\left(t^{\prime}\right)>1$. Consider such an interval $t^{\prime}$ together with an interval $t \in M$ with $|P(t)|=k$ and $P\left(t^{\prime}\right) \neq P(t)$. Then there is a player $i$ such that $i \in P\left(t^{\prime}\right)$ and $i \notin P(t)$. Combining the result of the claim with (4) implies that

$$
\beta_{N}(t)-\beta_{\min }(t)=\sum_{j=1}^{k-1} \beta_{(j)}(t)=1
$$

which shows $(c)$.

For the implication $(i i) \Rightarrow(i)$, suppose that the claim profile satisfies the conditions of $(i i)$. It is sufficient to check that players have no incentive to deviate from intervals $t \in M$ with $|P(t)|=k$ (since for all other intervals the marginal loss equals 1 , as $\beta_{N}(t)=1$ ). Due to Lemma 5.7 it is not profitable to deviate within the same interval, as the loss of removing $\Delta$ is at least $\frac{k-1}{k} \Delta$ (since the losses are equally divided) and the gain of placing it elsewhere is at most $\frac{k-1}{k} \Delta$. By similar arguments, it follows that there is also no incentive do deviate to an interval with a smaller or equal number of claimants.

So the only interesting case arises if there is a player $i \in P(t), i \notin P\left(t^{\prime}\right)$ where $t^{\prime} \in M$ with $\left|P\left(t^{\prime}\right)\right|=k$. As the minimum average loss on $t$ equals $\frac{k-1}{k}$, the most profitable claim to put on $t^{\prime}$ is the claim which assures that each player $j \in P\left(t^{\prime}\right)$ with $\beta_{j}\left(t^{\prime}\right)=\beta_{\min }\left(t^{\prime}\right)$ gets a share of zero. An additional increase in claim results in a marginal gain of at most $\frac{k-1}{k}$ and is thus not profitable. A lower claim does not take the marginal gain of $\frac{k}{k+1}$ into account, which means that the claim is not the most profitable one. More precisely, the claim needs to have a size of $k \beta_{\min }\left(t^{\prime}\right)$, as then $\mu=\beta_{\min }(t)$. The total gain on $t^{\prime}$ then equals $(k-1) \beta_{\min }\left(t^{\prime}\right)$, whereas the total loss is at least $k \frac{k-1}{k} \beta_{\min }\left(t^{\prime}\right)$. This proves that no profitable deviation between intervals is possible.

The following corollary presents the existence condition for an NEP.

Corollary 5.10. The game $\left(E, c, f^{C E L}\right)$ has an $N E P$ if and only if $\sum_{i \in N} c_{i} / 2 \leq E$. 
Proof. Let $(y, \beta, m)$ be an NEP. If $|P(t)|=1$, then $\beta_{N}(t)=1$. If $|P(t)| \geq 2$, we have by Lemma 5.7 that $\beta_{N}(t)-\beta_{\min }(t) \leq 1$ for all $t \in M$. So $|P(t)| \beta_{\min }(t)-\beta_{\min }(t) \leq \beta_{N}(t)-\beta_{\min }(t) \leq 1$, which implies that $\beta_{\min }(t) \leq \frac{1}{|P(t)|-1}$ and thus $\beta_{N}(t) \leq 1+\frac{1}{|P(t)|-1} \leq 2$. Together this implies that $\sum_{i \in N} c_{i}=\sum_{t \in M} \beta_{N}(t) \leq 2 E$.

Suppose $\sum_{i \in N} c_{i} / 2 \leq E$. We construct an NEP in the following way: every player puts a claim of 0 or 1 on every interval, and the total claim on every interval is 1 or 2 . If we distribute the entitlements iteratively from left to right and start again on the left for the second claims, then one can check that the claims profile satisfies the conditions of Theorem 5.9.

Remark 5.11. We can also use Lemma 5.7 to derive an upper bound on the number of claimants on an interval in an equilibrium as in Corollary 5.10. If $\sum_{i \in N} c_{i}=E$, then $\beta_{N}(t)=1$ for all $t \in M$ in equilibrium, and only $n$ is an upper bound. Now let $\ell \in \mathbb{R}, \ell>1$ be such that $\left(1+\frac{1}{\ell}\right) E<\sum_{i \in N} c_{i} \leq 2 E$. Then $\ell$ is an upper bound, which can be seen as follows. If $|P(t)|=1$, then clearly $|P(t)|<\ell$. If $|P(t)| \geq 2$, we have by Lemma 5.7 that $\beta_{N}(t) \leq 1+\beta_{\min }(t) \leq 1+\frac{1}{|P(t)|-1}$ for all $t \in M$. This implies that

$$
\left(1+\frac{1}{\ell}\right) E<\sum_{i \in N} c_{i}=\sum_{t \in M} \beta_{N}(t) \leq\left(1+\frac{1}{|P(t)|-1}\right) E,
$$

which in turn implies $|P(t)| \leq \ell$.

In order to be able to describe the equilibrium payoffs, we show that in equilibrium any interval can be redistributed such that the payoffs for the players remain unchanged and only intervals with one or two claimants are used.

Lemma 5.12. For every $N E P$ in $\left(E, c, f^{C E L}\right)$ there exists a payoff-equivalent $N E P$ such that $|P(t)| \leq 2$ and $\beta_{i}(t)=1$ for all $t \in M$ and $i \in P(t)$.

Proof. Let $(y, \beta, m)$ be an NEP. We will show that we can redistribute the claims on every interval $t \in M$ such that $\left|P\left(t^{\prime}\right)\right| \leq 2$ and $\beta_{i}\left(t^{\prime}\right)=1$ for all $i \in P\left(t^{\prime}\right)$, for every subinterval $t^{\prime}$ of $t$, but without changing a player's total share of the interval $t$. This generates a new finer claim profile which is still an NEP.

We only need to consider intervals $t \in M$ with $|P(t)| \geq 2$, since $\beta_{i}(t)=1$ for $i \in P(t)$ if $|P(t)|=1$. By Lemma 5.7, every interval $t$ with $|P(t)| \geq 2$ satisfies the following inequality: $1-\beta_{\min }(t) \leq \beta_{N}(t)-\beta_{\min }(t) \leq 1$. The share of each player $i \in P(t)$ for such an interval is

$$
f_{i}(\beta(t))=\beta_{i}(t)-\frac{\beta_{N}(t)-1}{|P(t)|} .
$$

We show that for some $\alpha$ with $y_{t-1}<\alpha<y_{t}$, we can reshuffle all the claims on $\left(y_{t-1}, y_{t}\right)$ such that on each subinterval of $\left(y_{t-1}, \alpha\right)$ there is a claim of 1 by two players, on each subinterval of $\left(\alpha, y_{t}\right)$ there is a claim of 1 by one player, and all shares of players $i \in P(t)$ remain unchanged. The procedure we use here is similar to the way we describe the payoffs in Corollary 3.3, using the vector $r$, only now applied to the specific interval $t$. Since $\beta_{N}(t)\left(y_{t}-y_{t-1}\right)=2\left(\alpha-y_{t-1}\right)+\left(y_{t}-\alpha\right)$, we have $\alpha=y_{t-1}+\left(\beta_{N}(t)-1\right)\left(y_{t}-y_{t-1}\right)$.

Let $x_{i}=\frac{2\left(\beta_{N}(t)-1\right)}{|P(t)|}$ for $i \in P(t)$ denote the part of the claim of player $i$ distributed on $\left(y_{t-1}, \alpha\right)$ such that on every part there is a claim of 1 by two players, and let $\beta_{i}(t)-x_{i}$ be distributed on $\left(\alpha, y_{t}\right)$ such that on every part there is a claim of 1 by one player.

In order to see that we have a feasible redistribution, note the following properties: 
(i) $x_{i}=\frac{2\left(\beta_{N}(t)-1\right)}{|P(t)|} \geq 0$.

(ii) $x_{i}=\frac{2\left(\beta_{N}(t)-1\right)}{|P(t)|} \leq \beta_{N}(t)-1 \leq \beta_{\min }(t) \leq \beta_{i}(t)$, where the first inequality follows since $|P(t)| \geq 2$ and the second inequality follows from Lemma 5.7.

(iii) $\sum_{i \in P(t)} x_{i}=2\left(\beta_{N}(t)-1\right)$.

Moreover, the share of player $i \in P(t)$ is

$$
\frac{1}{2} x_{i}+\beta_{i}(t)-x_{i}=\beta_{i}(t)-\frac{1}{2} x_{i}=\beta_{i}(t)-\frac{\beta_{N}(t)-1}{|P(t)|}=f_{i}(\beta(t)) .
$$

If we reshuffle every interval in the above way, we end up with an equilibrium claim profile without changing the shares of the players.

Lemma 5.12 makes it possible to describe the payoffs associated with NEPs: all these payoffs can be found by only using intervals with either one or two claimants. Hence the set of payoff vectors is equal to the set found in Corollary 3.3.

\section{$6 \quad$ Unrestricted problems}

In this section we discuss NEPs for unrestricted problems. That is, players $i \in N$ may have entitlements with $c_{i}>E$.

\subsection{Constrained equal awards}

If the constrained equal awards rule is used as sharing rule and we assume integer claim heights, then we obtain the following lemma, which says that in equilibrium every player with an entitlement of at least the estate, puts a claim of 1 on the entire estate.

Lemma 6.1. Let $(y, \beta, m)$ be an NEP. If $c_{i} \geq E$ for player $i$, then $\beta_{i}(t) \geq 1$ for all $t \in M$.

Proof. Let $(y, \beta, m)$ be an NEP. Suppose, to the contrary, that player $i$ does not claim interval $t \in M$. Since $c_{i} \geq E$, there exists an interval $t^{\prime} \in M$ with $\beta_{i}\left(t^{\prime}\right) \geq 2$. The net gain of removing a claim amount of 1 from $t^{\prime}$ and putting it on (a part of) $t$ is positive, which contradicts that $(y, \beta, m)$ is an NEP.

Hence, under integer claims, every player $i$ with $c_{i} \geq E$ claims the estate once and is indifferent where to put his remaining claims, as this remainder does not affect his nor his opponents' payoffs. So in fact, we are allowed to ignore the part of the entitlement that is above the amount of the estate without changing the equilibrium outcome. Therefore, we can solve the unrestricted problem as a restricted problem where the entitlement of every player $i$ with $c_{i} \geq E$ is equal to $E$. For the analysis of these problems see Subsection 4.1. We obtain the following theorem.

Theorem 6.2. Under integer claims, $U\left(E, c, f^{C E A}\right)=U\left(E, c^{\prime}, f^{C E A}\right)$, where $c_{i}^{\prime}=\min \left\{c_{i}, E\right\}$ for all $i \in N$.

Also if arbitrary claims are allowed, both the restricted and unrestricted problems result in the same analysis: all NEPs result in the same payoffs, equal to the payoffs assigned by the constrained equal awards rule. Hence, Theorem 5.3 still applies. 


\subsection{Talmud rule}

If the Talmud rule is used as sharing rule in the game with integer claims, then recall that this rule is equal to the constrained equal awards sharing rule. Thus, the same analysis applies.

In case of arbitrary claims, we obtain the following theorem.

Theorem 6.3. If there is a player $i$ with $c_{i}>E$, then all NEPs result in the same payoffs, equal to the payoffs assigned by the Talmud rule.

Proof. Note that if $\sum_{i \in N} c_{i} / 2>E$, the presence or absence of a player $i$ with $c_{i}>E$ does not make a difference for the analysis in Theorem 5.4. Hence this result still holds.

If $\sum_{i \in N} c_{i} / 2 \leq E$, then a player $i$ with $c_{i}>E$ puts a claim of 1 on the entire estate, so that the share of each player $j \in N \backslash\{i\}$ is at most $\frac{1}{2} \beta_{j}(t)$ for every $t \in M$. Since $\sum_{j \in N} c_{j} \leq 2 E$, every player $j \in N \backslash\{i\}$ is able to assure a share of $\frac{1}{2} \beta_{j}(t)$ on all $t \in M$, so that in equilibrium every player $j \in N \backslash\{i\}$ receives $\frac{1}{2} c_{j}$ and $i$ receives $E-\sum_{i \in N \backslash\{i\}} \frac{1}{2} c_{i}$. This is precisely what every player obtains if the Talmud rule is applied to the estate division problem.

\subsection{Constrained equal losses}

If the constrained equal losses rule is used as sharing rule, the main observation is that no NEP exists if there is some player $i$ with $c_{i}>E$, independent of whether we consider integer or arbitrary claims.

Theorem 6.4. If there is a player $i$ with $c_{i}>E$, then the game $\left(E, c, f^{C E L}\right)$ with integer or with arbitrary claims has no NEP.

Proof. We first prove the statement for integer claims and afterwards for arbitrary claims.

Let player $i$ have $c_{i}>E$, and suppose that $(y, \beta, m)$ is an NEP in the game with integer claims. We derive a contradiction. It can be checked that Lemmas 4.6 and 4.7 also apply for unrestricted problems, which implies that in equilibrium $\beta_{j}(t) \leq 1$ for all $j \in N$ and all $t \in M$. However, since $c_{i}>E$ for player $i$, there must exist some interval $t \in M$ with $\beta_{i}(t)>1$. This is a contradiction.

Next, let again player $i$ have $c_{i}>E$, and suppose that $(y, \beta, m)$ is an NEP in the game with arbitrary claims. We again derive a contradiction. Note that $\beta_{N}(t)=1$ if $|P(t)|=1$, as a free claim can always be used to gain a positive amount somewhere else. Lemma 5.7 (which can be seen to hold also for unrestricted problems) implies that $\beta_{j}(t) \leq 1$ for all $j \in N$ and all $t \in M$ with $|P(t)| \geq 2$. However, since $c_{i}>E$ for player $i$, there must exist some interval $t \in M$ with $\beta_{i}(t)>1$. This is a contradiction.

\section{Summary and conclusion}

We have analyzed the estate division problem as a non-cooperative game, in which every player uses his entitlement to claim specific parts of the estate. Every part is then distributed based on these integer valued or arbitrary claims, according to a sharing rule. We have investigated the payoffs associated with the equilibrium outcomes of this game. Our first main result is an axiomatic result for restricted claim games with $\sum_{i \in N} c_{i} / 2 \leq E$. We characterize the set of equilibrium payoffs under four fairly general axioms and show that all rules always resulting in an equilibrium payoff

satisfy minimal rights first and lower bound of degree half. Examples are the Talmud rule, the adjusted proportional rule and the random arrival rule. 


\begin{tabular}{|c|c|c|c|c|}
\hline & $\begin{array}{l}\text { Restricted and } \\
\text { integer claims }\end{array}$ & $\begin{array}{c}\text { Restricted, } \\
\text { arbitrary claims and } \\
\sum_{i \in N} c_{i} / 2 \leq E\end{array}$ & $\begin{array}{c}\text { Restricted, } \\
\text { arbitrary claims and } \\
\sum_{i \in N} c_{i} / 2>E\end{array}$ & $\begin{array}{l}\text { Unrestricted and } \\
\text { integer claims }\end{array}$ \\
\hline$f^{C E A}$ & $\begin{array}{l}v_{i}=\frac{c_{i}}{k}-\frac{r_{i}}{k(k+1)} \\
(\text { Subsection } 4.1)\end{array}$ & $\begin{array}{l}\text { CEA-payoff } \\
\text { (Theorem 5.3) }\end{array}$ & $\begin{array}{l}\text { CEA-payoff } \\
\text { (Theorem 5.3) }\end{array}$ & $\begin{array}{c}\text { Solve }\left(E, c^{\prime}, f^{C E A}\right), \\
\text { where } c_{i}^{\prime}=\min \left\{c_{i}, E\right\} \\
\quad(\text { Theorem } 6.2)\end{array}$ \\
\hline$f^{T}$ & $\begin{array}{c}v_{i}=\frac{c_{i}}{k}-\frac{r_{i}}{k(k+1)} \\
(\text { Lemma } 4.11)\end{array}$ & $\begin{array}{c}v_{i}=c_{i}-\frac{r_{i}}{2} \\
(\text { Lemma } 5.5)\end{array}$ & $\begin{array}{l}\text { Talmud-payoff } \\
\text { (Theorem 5.4) }\end{array}$ & $\begin{array}{c}\text { Solve }\left(E, c^{\prime}, f^{T}\right), \\
\text { where } c_{i}^{\prime}=\min \left\{c_{i}, E\right\}\end{array}$ \\
\hline$f^{C E L}$ & $\begin{array}{c}v_{i}=c_{i}-\frac{r_{i}}{2} \\
\text { (Corollary 3.3) }\end{array}$ & $\begin{array}{c}v_{i}=c_{i}-\frac{r_{i}}{2} \\
(\text { Lemma } 5.12)\end{array}$ & $\begin{array}{c}\text { No NEPs } \\
(\text { Corollary 5.10) }\end{array}$ & $\begin{array}{c}\text { No NEPs } \\
\text { (Corollary 6.4) }\end{array}$ \\
\hline
\end{tabular}

Table 1: A summary of the equilibrium payoffs for the three main sharing rules for different problems. For clarification, we refer to the corresponding references.

Then we turn to claim games with sharing rules derived from the TAL-family. These results are summarized in Table 1. The first (most left) column describes the possible NEP payoffs in integer claim games associated with restricted problems. The payoff from the CEL sharing rule arises from taking $k=1$ : NEPs exist if and only if $\sum_{i \in N} c_{i} \leq 2 E$. For all restricted problems $(E, c) \in \mathcal{C}^{N}$, we have the following relation between the different sets of equilibrium payoffs:

$$
U\left(E, c, f^{C E L}\right) \subseteq U\left(E, c, f^{C E A}\right)=U\left(E, c, f^{T}\right) \subseteq U\left(E, c, f^{P}\right) .
$$

The second column maintains the condition $\sum_{i \in N} c_{i} \leq 2 E$, but allows for arbitrary claims. This does not essentially affect the payoffs under CEL or Talmud, but for CEA all payoffs coincide with the payoffs assigned by the CEA rule to the original estate division problem. The third column concerns arbitrary claims under the condition $\sum_{i \in N} c_{i} / 2>E$. The fourth column collects some results for unrestricted problems.

In future research, the present analysis may be extended to still other rules, e.g., the ICI (containing the TAL-family) and CIC classes in Thomson (2008). An interesting open question is whether a rule exists that results in an equilibrium payoff for problems with $\sum_{i \in N} c_{i} / 2>E$.

\section{References}

Atlamaz, M., Berden, C., Peters, H., Vermeulen, D., 2011. Non-cooperative solutions for estate division problems. Games and Economic Behavior 73, 39-51.

Aumann, R., Maschler, M., 1985. Game theoretic analysis of a bankruptcy problem from the Talmud. Journal of Economic Theory 36, 195-213.

Berliant, M., Thomson, W., Dunz, K., 1992. On the fair division of a heterogeneous commodity. Journal of Mathematical Economics 21, 201-206.

Borel, E., 1921. La Théorie du Jeu et les Équations Intégrales à Noyau Symétrique. Comptes Remdus de l'Académie des Sciences 173, 1304-1308. Translated by Savage, L.J.,1953. The theory of play and integral equations with skew symmetric kernels. Econometrica 21, 97-100. 
Cramton, P., Gibbons, R, Klemperer, P., 2003. Dissolving a partnership efficiently. Econometrica $55,615-632$.

Curiel, I., Maschler, M., Tijs, S.H., 1987. Bankruptcy games. Zeitschrift für Operations Research 31, A143-A159.

Chun, Y., Schummer, J., Thomson, W., 2001. Constrained egalitarianism: a new solution to bankruptcy problems. Seoul Journal of Economics 14, 269-297.

Hotelling, H., 1929. Stability in competition. The Economic Journal 39, 41-57.

Merolla, J., Munger, M., Tofias, M., 2005. In play: a commentary on strategies in the 2004 US presidential election. Public Choice 123, 19-37.

Moreno-Ternero, J., Villar, A., 2006. The TAL-family of rules for bankruptcy problems. Social Choice and Welfare 27,231-249.

Moreno-Ternero, J., 2011. A coalitional procedure leading to a family of bankruptcy rules. Operations Research Letters 39, 1-3.

O'Neill, B., 1982. A problem of rights arbitration from the Talmud. Mathematical Social Sciences $2,345-371$.

Pálvölgyi, D., Peters, H., Vermeulen, D., 2013. A strategic approach to estate division problems with non-homogeneous preferences. Maastricht University. (An earlier version appeared as METEOR Research Memorandum 10/036, Maastricht, 2010.)

Thomson, W., 2003. Axiomatic and game-theoretic analysis of bankruptcy and taxation problems: a survey. Mathematical Social Sciences 45, 249-297.

Thomson, W., 2008. Two families of rules for the adjudication of conflicting claims. Social Choice and Welfare 31, 667-692. 